\title{
Nature and Control of Shakeup Processes in Colloidal Nanoplatelets
}

\author{
Jordi Llusar and Juan I. Climente* \\ Departament de Química Física i Analítica, Universitat Jaume I, E-12080, Castelló de la \\ Plana, Spain \\ E-mail: climente@uji.es
}

\begin{abstract}
Recent experiments suggest that the photoluminescence line width of CdSe nanoplatelets (NPLs) and core/shell CdSe/CdS NPLs may be broadened by the presence of shakeup (SU) lines from negatively charged trions. We carry out a theoretical analysis, based on effective mass and configuration interaction (CI) simulations, to identify the physical conditions that enable such processes. We confirm that trions in colloidal NPLs are susceptible of presenting $\mathrm{SU}$ lines up to one order of magnitude stronger than in epitaxial quantum wells, stimulated by dielectric confinement. For these processes to take place, trions must be weakly bound to off-centered charge traps, which relax symmetry selection rules. Charges on the lateral sidewalls are particularly efficient to this end. Our simulations display a single strong SU replica in most instances, which agrees well with experiments on CdSe NPLs, but suggests that the multi-peaked emission reported for core/shell CdSe/CdS NPLs must involve other factors beyond SU processes. We propose emission from a metastable spin triplet trion state may be responsible. Understanding the origin of SU processes may open paths to rational design of NPLs with narrower line width.
\end{abstract}




\section{Keywords}

trion emission, radiative Auger, heterostructure, impurities, CI method

Colloidal metal chalcogenide NPLs offer well defined advantages over their quantum dot and rod counterparts as semiconductor building blocks for optical applications. ${ }^{1-4}$ Some of the most distinctive features are order-of-magnitude shorter radiative lifetimes, which result from the strong exciton binding energies in quasi-2D systems (Giant Oscillator Strength effect), ${ }^{5,6}$ and precisely controlled thickness of the nanostructure, ${ }^{7-10}$ which largely suppresses the emission broadening due to size dispersion usually observed in dots. These properties give rise to bright and narrow emission lines, which are of interest for displays, lighting and lasers. ${ }^{3,4}$

Unfortunately, ligand passivation of NPL surface dangling bonds is usually incomplete because of labile binding and steric hindrance between ligands. This can translate into significant non-radiative losses. ${ }^{11}$ To overcome this problem, core-only NPLs are sometimes replaced by core/shell or core/crown heterostructures, where some facets of the core material are coated with a higher band gap inorganic material. ${ }^{1}$ Typical heterostructures are $\mathrm{CdSe} / \mathrm{CdS},{ }^{12-14} \mathrm{CdSe} / \mathrm{CdTe},{ }^{15,16} \mathrm{CdSe} / \mathrm{ZnS}^{17,18}$ and their alloys. ${ }^{19,20}$ These heterostructures succeed in isolating the photogenerated carriers, which remain in and around the core, from the NPL surfaces, thus translating into enhanced fluorescence quantum efficiency and photostability. ${ }^{1,4,21}$ The heterostructure growth has however a negative side effect, namely the systematic broadening of the emission line width, e.g. from $\sim 35-40 \mathrm{meV}$ in CdSe NPLs to $\sim 60-80 \mathrm{meV}$ in core/shell CdSe/CdS NPLs. ${ }^{12,22}$ Line width broadening in such NPLs was initially ascribed to the presence of local traps induced upon shell coating. ${ }^{12}$ Graded interface composition was then used to narrow the line width down to $\sim 55 \mathrm{meV},{ }^{19,20}$ but this figure is still larger than in core-only NPLs, which suggests that interface defects are not the only source of broadening.

To shed light into this problem, Antolinez and co-workers recently investigated the origin of the fluorescence line width broadening in core/shell CdSe/CdS NPLs by means of 
single-particle spectroscopy. ${ }^{23}$ They observed that individual NPLs present a series of 2 to 4 narrow peaks split from each other by $\sim 10 \mathrm{meV}$. Altogether, the peaks fit well the asymmetric line shape of ensemble NPLs at cryogenic temperatures. ${ }^{12}$ A similar feature was soon after reported in core-only CdSe NPLs, although in this case only two peaks were measured. ${ }^{24}$ Having ruled out more conventional mechanisms, such as exciton-phonon interaction or spectral diffusion, the nature of the additional peaks was tentatively ascribed to SU processes of negative trions $\left(\mathrm{X}^{-}\right)$. These are partly radiative Auger processes, whereby an electronhole pair recombines radiatively but transfers part of its energy to the remaining electron by exciting it into a higher single-electron level (in-plane excitation). They have been previously reported in epitaxial quantum wells ${ }^{25-28}$ and self-assembled quantum dots ${ }^{29}$ under the magnetic fields, corresponding to inter-Landau level excitations of the excess carrier. ${ }^{30}$ More recently, SU peaks have been clearly revealed in the magneto-photoluminescence of electrically charged InGaAs and GaAs quantum dots. ${ }^{31}$ By contrast, they do not seem to appear in type-II core/crown NPLs. ${ }^{32}$ In this context, clarifying the role of SU processes in the emission of colloidal NPLs is a desirable step to better understand and control the emission line width of NPLs, which would be advantageous for optical applications.

In this work, we analyze the possible occurence of SU processes in colloidal CdSe-based NPLs from a theoretical perspective. The goal is to determine which physical conditions enable these processes. To this end we use effective mass models and full CI simulations, which provide an intuitive description of the underlying physics. We shall confirm that one intense SU replica can be expected for negative trions $\left(X^{-}\right)$in both core-only and core/shell NPLs, corresponding to the excitation of the remaining electron into a higher orbital with the same symmetry as the ground state. For this to take place, the trion must be weakly bound to an off-centered acceptor charge. The role of the charge is to lower the system symmetry, thus relaxing selection rules, and to stimulate electron-electron repulsion (quench electron-hole attraction) in the ground orbital. By doing so, SU peaks can reach intensities exceeding $10 \%$ of the fundamental (band edge, fully radiative) transition. This is one order of magnitude 
higher than in epitaxial quantum wells, which can be rationalized from the stronger Coulomb interactions in NPLs, which result from the pronounced dielectric confinement, and from the presence of lateral sidewalls, which are prone to surface traps. Analogous conclusions hold for positive trions. We discuss connections with experiments in the literature and propose potential strategies to suppress these processes.

\section{Results}

We analyze the emission spectra of trions in core-only and core/shell NPLs. Negative trions are studied unless otherwise noted, as it is the most frequently reported species in these structures, ${ }^{23,24,33,34}$ but the conclusions do not depend on the sign of the charged exciton (see Fig. S2 in the Supporting Information, SI). Once the general behavior of SU processes in these systems is understood, we discuss how our conclusions fit the interpretation of different experimental observations and the practical implications of our findings.

\section{Core-only NPLs}

We start by studying core-only CdSe NPLs. The NPLs are chosen to have 4.5 monolayer (ML) thickness and a lateral size of $20 \times 20 \mathrm{~nm}^{2}$, for similarity to the core dimensions of

Ref. ${ }^{23}$ They have a pronounced dielectric mismatch with the organic environment, which we model with $\epsilon_{\text {in }}=6$ and $\epsilon_{\text {out }}=2$ as the dielectric constants inside and outside the NPL, unless otherwise stated. ${ }^{35,36}$ The presence of few-meV spectral jumps in photoluminescence experiments $^{23}$ suggests that the trion is subject to the influence of carriers temporarily trapped on the surface. ${ }^{22,37}$ To model this phenomenon, a fractional point charge is placed on the surface, with charge $Q=e Q_{X}\left(\left|Q_{X}\right| \leq 1\right.$ and $e$ is the full electron charge). The fractional value of $Q_{X}$ accounts for the screening of trapped charges (e.g. a hole) by the trap defect itself (e.g. a surface dangling bond). ${ }^{38}$ Two scenarios are considered: a charge centered on the top facet $\left(Q_{t o p}\right)$ and an off-centered charge, located along the edge of a 
lateral facet $\left(Q_{\text {edge }}\right)$. The latter setup is suggested by studies showing that edge and vertex atoms in CdSe structures have weaker binding to oleate ligands. ${ }^{39}$ The two systems are represented in Figure 1a and 1b. The corresponding emission spectra are shown in Fig. 1c and $1 \mathrm{~d}$. The figure reveals a number of important observations. (i) In the absence of surface charge $\left(Q_{X}=0\right.$, thick lines), only the fundamental transition shows up, with no sizable SU replica. (ii) Charges on the top facet induce SU peaks (see arrow in Fig. 1c), but their strength is two orders of magnitude smaller than that of the fundamental transition (main line). This is similar to the case of epitaxial quantum wells. ${ }^{25-28}$ (iii) Stronger SU replica are however obtained for charges located on the lateral sidewall, provided the charge is attractive (acceptor charge) and binding to the trion is moderately weak, see Fig. 1d. For $Q_{\text {edge }}=0.4$ (marked with a star in the figure), the SU peak reaches $\sim 25 \%$ of the main peak height. This ratio is about 20 times higher than in epitaxial quantum wells, and it holds despite the Giant Oscillator Strength enhancing the band edge recombination, ${ }^{5-7,36}$ which suggests that $\mathrm{SU}$ satellites also benefit from this phenomenon. For $Q_{\text {edge }}>0.4$, however, the SU peak intensity is lowered again and the energy splitting (redshift) with respect to the main line increases. Second and third SU lines are built for strong surface charges (see inset in Fig. 1d at $\left.Q_{\text {edge }}=0.7\right)$, but their magnitude is negligible. We have also explored different locations of the charge, obtaining intermediate results between those shown in Fig. 1 (see Fig. S3 in SI). These results point out the potentially significant role of lateral sidewalls, which are characteristic feature of colloidal quantum wells as compared to epitaxial ones, in obtaining high SU peaks.

To gain understanding on the origin of strong SU peaks when trions bind to lateral surface acceptors, beyond the full numerical calculation of Fig. 1, in Fig. 2a and 2b we compare sketches of the $\mathrm{SU}$ processes, in the absence and presence of an attractive edge charge. Within effective mass theory, the conduction band and valence band energy levels of (non-interacting) electrons and holes can be described as particle-in-the-box states, with quantum numbers $\left(n_{x}, n_{y}, n_{z}\right)$. It is useful however to label the states by their symmetry 
a)

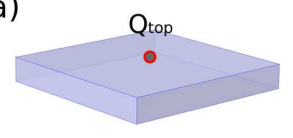

b)
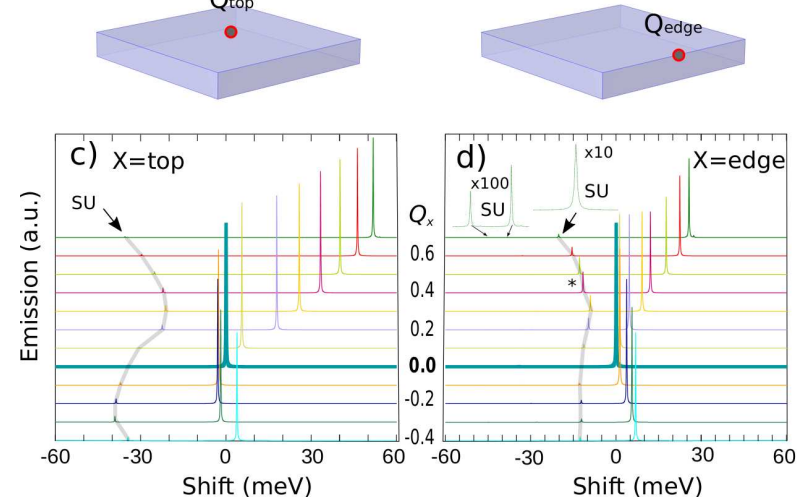

Figure 1: (a,b) Schematics of core-only NPLs with different location of the surface charge. (c,d) Corresponding $\mathrm{X}^{-}$emission spectrum for charge strength $Q=Q_{X} e$. The arrows point at the SU satellites (shaded lines are guides to the eyes). The highest $\mathrm{SU}$ peak is observed for off-centered acceptor charges weakly bound to the trion $\left(Q_{\text {edge }}=0.4\right.$, marked with a star in (d)). The spectra are normalized to the intensity of the fundamental transition at $Q_{X}=0$, and offset vertically for clarity. The insets for $Q_{e d g e}=0.7$ in (d) show amplified SU peaks.

(irreducible representation). When $Q_{\text {edge }}=0$, because the NPL has squared shape, the point group is $D_{4 h}$. When $Q_{\text {edge }} \neq 0$, the electrostatic potential yields a symmetry descent to $C_{s}$. As a consequence, degeneracies are lifted and additional states with the same symmetry as the ground orbital $\left(A^{\prime}\right)$ are obtained. This is important because after electron-hole recombination, the excess electron can only be excited to an orbital with the same symmetry as the initial one (as shown by the vertical arrows in Fig. 2a and 2b). Therefore, lowering the system symmetry opens new channels for SU processes. Furthermore, these can involve low-energy orbitals, which have fewer nodes and hence larger overlap with the trion ground state, as we shall see below. Both the number and the intensity of the SU processes are in principle enhanced. By contrast, a centered charge on the top surface barely affects the system symmetry, which remains high $\left(C_{4 v}\right)$, and SU processes are only slightly stronger than in the $Q_{\text {edge }}=0$ case.

The qualitative reasoning above can be substantiated with a CI formalism on the basis of independent particle (non-interacting) electron and hole states, ${ }^{31}$ which has the additional advantage of giving intuitive insight on how Coulomb interactions affect the likelihood of SU 


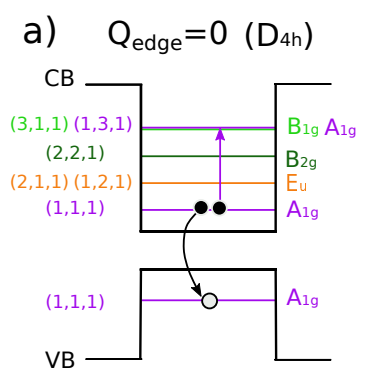

c)

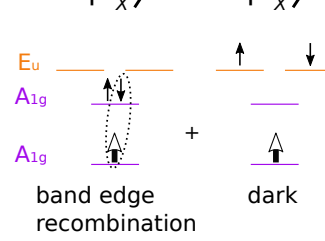

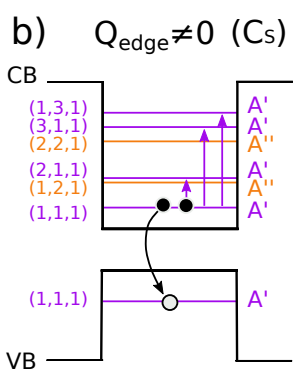

d) $\left|1_{x}\right\rangle \quad\left|2_{x}\right\rangle$

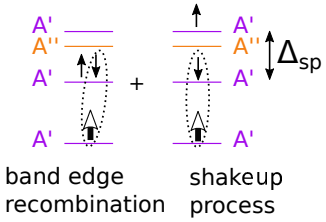

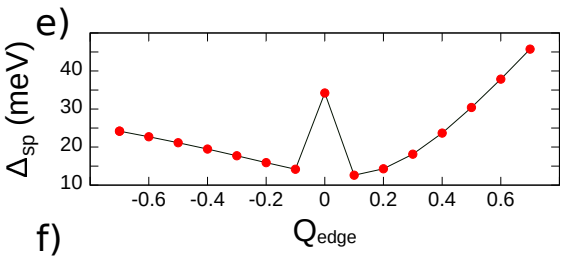

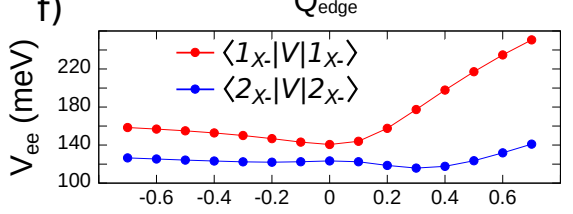

g)

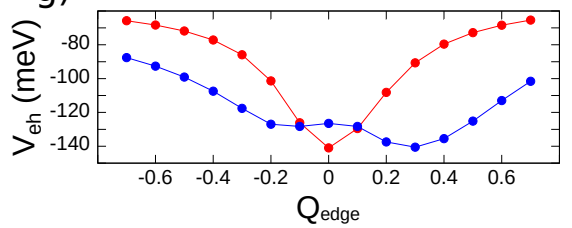

Figure 2: (a,b) Sketch of SU processes in NPLs with (a) and without (b) an edge charge. Labels on the left are $\left(n_{x}, n_{y}, n_{z}\right)$ quantum numbers for the (independent particle) energy levels. Labels on the right are the corresponding irreducible representation. The surface charge lowers the point group symmetry, from $D_{4 h}$ to $C_{s}$, lifting degeneracies and enabling new channels for SU transitions (vertical arrows). (c,d) Two main configurations $\left|m_{X^{-}}\right\rangle$in the CI expansion of $\left|G S_{X^{-}}\right\rangle$, with and without edge charge. Thin (thick) arrowsheads denote electron (hole) spin. Only when $Q_{e d g e} \neq 0$ a SU process is expected. (e) Energy splitting between $\left|1_{X^{-}}\right\rangle$and $\left|2_{X^{-}}\right\rangle$at an independent particle level. (f) average value of electronelectron repulsion and (g) electron-hole attraction in configurations $\left|1_{X^{-}}\right\rangle$and $\left|2_{X^{-}}\right\rangle$. 
processes. We consider that the transition rate from the trion ground state $\left|G S_{X^{-}}\right\rangle$to an electron spin-orbital $\left|f_{e}\right\rangle$, is proportional to: ${ }^{40}$

$$
P_{G S \rightarrow f}=\left|\left\langle f_{e}|\hat{P}| G S_{X^{-}}\right\rangle\right|^{2}
$$

$\hat{P}$ is the dipolar transition operator, $\hat{P}=\sum_{i_{e}, i_{h}}\left\langle i_{e} \mid i_{h}\right\rangle e_{i_{e}} h_{i_{h}}$, where $e_{i_{e}}$ and $h_{i_{h}}$ are annihilation operators for independent electron and hole spin-orbitals $\left|i_{e}\right\rangle$ and $\left|i_{h}\right\rangle$, respectively. We describe the trion ground state with a CI expansion,

$$
\left|G S_{X^{-}}\right\rangle=\sum_{m} c_{m}\left|m_{X^{-}}\right\rangle
$$

where $\left|m_{X^{-}}\right\rangle$is a trion configuration: $\left|m_{X^{-}}\right\rangle=e_{r_{e}}^{\dagger} e_{s_{e}}^{\dagger}|0\rangle_{e} h_{t_{h}}^{\dagger}|0\rangle_{h}$, with $e_{r_{e}}^{\dagger}$ and $h_{t_{h}}^{\dagger}$ creator operators, $|0\rangle_{e}$ and $|0\rangle_{h}$ the vacuum occupation vectors of electron and hole, and $c_{m}$ the coefficient in the expansion. Inserting $\hat{P}$ and $\left|G S_{X^{-}}\right\rangle$into Equation (1), one obtains:

$$
P_{G S \rightarrow f}=\left|\sum_{m} c_{m}\left(\left\langle r_{e} \mid t_{h}\right\rangle \delta_{f_{e} s_{e}}-\left\langle s_{e} \mid t_{h}\right\rangle \delta_{f_{e} r_{e}}\right)\right|^{2} .
$$

In $\mathrm{SU}$ processes, $\left|f_{e}\right\rangle$ is an excited spin-orbital. It then follows from Equation (3) that such a transition will only take place if $\left|G S_{X^{-}}\right\rangle$contains at least one configuration $\left|m_{X^{-}}\right\rangle$in the CI expansion where one electron is in the excited spin-orbital and the other electron has finite overlap with the hole ground state $\left(\left|s_{e}\right\rangle=\left|f_{e}\right\rangle\right.$ and $\left\langle r_{e} \mid t_{h}\right\rangle \neq 0$ or $\left|r_{e}\right\rangle=\left|f_{e}\right\rangle$ and $\left.\left\langle s_{e} \mid t_{h}\right\rangle \neq 0\right)$. The larger the weight of this configuration, $\left|c_{m}\right|^{2}$, the more likely the SU process. It is worth noting that in the strong confinement limit, the trion ground state is well described by a single configuration where all carriers are in the lowest-energy spin-orbitals (configuration $\left|1_{X^{-}}\right\rangle$in Fig. $2 \mathrm{c}$ and $2 \mathrm{~d}$ ). That is, $c_{1} \approx 1$ and $c_{m} \approx 0$ for $m>1$. SU transitions are then forbidden, which is why SU peaks are rarely reported in nanocrystals. On the contrary, in systems where Coulomb interaction energies exceed quantum confinement energies, the CI expansion contains mono- and biexcitations of electrons. SU processes are then enabled. Colloidal 
NPLs constitute an ideal system at this regard, because they combine weak confinement in the lateral direction with strong Coulomb interactions. ${ }^{41,42}$ Hereafter, we refer to this condition $\left(c_{m} \neq 0\right.$ for $\left.m>1\right)$ as Coulomb admixture.

The role of Coulomb admixture and symmetry breaking in activating SU processes can be illustrated, in the simplest approximation, by considering the two lowest-energy configurations of the trion ground state,

$$
\left|G S_{X^{-}}\right\rangle \approx c_{1}\left|1_{X^{-}}\right\rangle+c_{2}\left|2_{X^{-}}\right\rangle
$$

In Fig. 2c and 2d we depict such configurations in the absence and presence of an edge charge, respectively. These can be expected to be the two most important configurations in the full CI expansion. Notice that the two configurations must have the same symmetry, for Coulomb interaction to couple them. Because the lowest-energy configuration, $\left|1_{X^{-}}\right\rangle$, is always totally symmetric, so must be $\left|2_{X^{-}}\right\rangle$. Thus, when $Q_{\text {edge }}=0$ ( $D_{4 h}$ group), the electronic configuration of $\left|1_{X^{-}}\right\rangle$is $\left[A_{1 g}^{2}\right]_{e}\left[A_{1 g}\right]_{h}$, and that of $\left|2_{X^{-}}\right\rangle$is $\left[E_{u}^{2}\right]_{e}\left[A_{1 g}\right]_{h}$. The recombination of the $E_{u}$ electrons with the hole, which stays in a $A_{1 g}$ orbital, is then symmetry forbidden $\left(\left\langle r_{e} \mid t_{h}\right\rangle=\right.$ $\left\langle s_{e} \mid t_{h}\right\rangle=0$ in Eq. (3)). By contrast, when $Q_{e d g e} \neq 0\left(C_{s}\right.$ group $),\left|2_{X^{-}}\right\rangle$is formed by a monoexcitation where one electron is placed in the $\left(n_{x}, n_{y}, n_{z}\right)=(2,1,1)$ orbital, which also has $A^{\prime}$ symmetry, resulting in an electronic configuration $\left[A^{\prime} A^{\prime}\right]_{e}\left[A^{\prime}\right]_{h}$ (see Fig.2d). The hole can then recombine with the ground orbital electron, as both have $A^{\prime}$ symmetry $\left(\left\langle r_{e} \mid t_{h}\right\rangle \neq 0\right.$ or $\left\langle s_{e} \mid t_{h}\right\rangle \neq 0$ in Eq. (3)) and leave the excited electron as the final state. This constitutes a SU process. Because both SU and fundamental transitions rely on the recombination of the same electron-hole pair (same overlap integral, e.g. $\left\langle r_{e} \mid t_{h}\right\rangle$ ), the ratio between SU and fundamental radiative rates can be approximated as:

$$
\frac{P_{G S \rightarrow(2,1,1)_{e}}}{P_{G S \rightarrow(1,1,1)_{e}}} \approx \frac{\left|c_{2}\right|^{2}}{\left|c_{1}\right|^{2}} .
$$

i.e. it is set exclusively by the degree of Coulomb admixture. 
One can guess the requirements that maximize $\left|c_{2}\right|^{2}$ by looking which conditions favor energetically $\left|2_{X^{-}}\right\rangle$over $\left|1_{X^{-}}\right\rangle$. These include: (i) small energy splitting between the two configurations, at an independent particle level, $\Delta_{s p}$ in Fig. 2d, (ii) weaker electron-electron repulsion $\left(V_{e e}\right)$ and (iii) stronger electron-hole attraction $\left(V_{e h}\right)$ in $\left|2_{X^{-}}\right\rangle$as compared to $\left|1_{X^{-}}\right\rangle$. Figures 2e-g show that these conditions are met for moderately attractive (positive) charges $\left(Q_{e d g e} \sim 0.3-0.4\right)$. When the off-centered charge is switched on, $\Delta_{s p}$ rapidly decreases (see Fig. 2e) because the symmetry descent turns one of the $E_{u}$ (p-like) electron orbitals into a $A^{\prime}$ (s-like) one. However, the surface charge brings about electrostatic confinement and hence $\Delta_{s p}$ increases again soon after. As for inter-electron repulsion, $\left\langle 1_{X^{-}}\left|V_{e e}\right| 1_{X^{-}}\right\rangle$ increases more rapidly than $\left\langle 2_{X^{-}}\left|V_{e e}\right| 2_{X^{-}}\right\rangle$(see Fig. 2f) because the former involves placing the two electrons in identical orbitals, while the latter does not. Last, $\left\langle 1_{X^{-}}\left|V_{e h}\right| 1_{X^{-}}\right\rangle$is rapidly quenched (see Fig. 2g) because it involves the ground orbitals of electron and hole $-(1,1,1)_{e}$ and $(1,1,1)_{h^{-}}$, which dissociate rapidly under an external charge. $\left\langle 2_{X^{-}}\left|V_{e h}\right| 2_{X^{-}}\right\rangle$ stays strong up to $Q_{e d g e} \sim 0.3$ because it involves the $(2,1,1)_{e}$ orbital, which is spatially more extended and then keeps significant overlap with the $(1,1,1)_{h}$ hole. Figs. 2e-f further evidence that $Q_{\text {edge }}>0.3-0.4$ is inconvenient for $\mathrm{SU}$ processes, because the electrostatic potential increases lateral quantum confinement ( $\Delta_{s p}$ increases) and because the electrons and hole in configuration $\left|2_{X^{-}}\right\rangle$are eventually dissociated as well $\left(\left\langle 2_{X^{-}}\left|V_{e h}\right| 2_{X^{-}}\right\rangle\right.$is quenched in Fig. 2g).

Many of the above observations can be visualized by analyzing the evolution of charge densities and wave functions under $Q_{\text {edge }}$. In Figure 3 we show the two-electron (first row) and one-hole (second row) charge densities of $\left|G S_{X^{-}}\right\rangle$, obtained from the CI calculations of Fig. 1. The wave functions of the two lowest electron orbitals which can constitute configuration $\left|2_{X^{-}}\right\rangle,-\left(n_{x}, n_{y}, n_{z}\right)_{e}=(1,1,1)_{e}$ and $(2,1,1)_{e^{-}}$are also plotted (bottom rows). At $Q_{\text {edge }} \approx 0$, the two orbitals are quasi-orthogonal. As a result, Coulomb interaction cannot couple configurations $\left|1_{X^{-}}\right\rangle$and $\left|2_{X^{-}}\right\rangle$, so that $c_{2} \approx 0$. This is why the two-electron charge density closely resembles the $(1,1,1)_{e}$ orbital. SU processes are not expected in this case. 


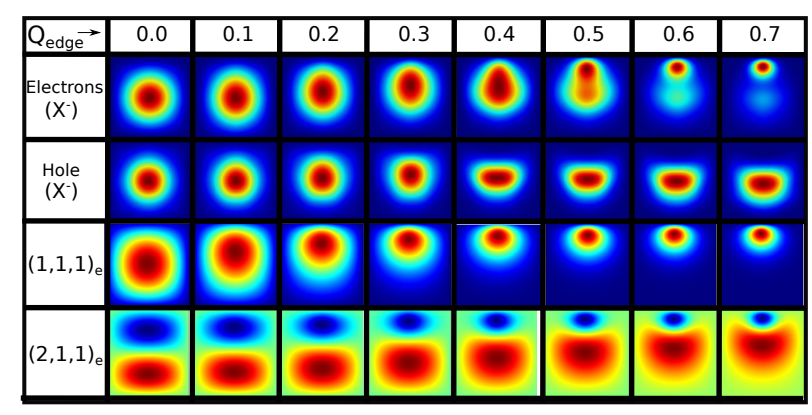

Figure 3: In-plane charge density of the two electrons and hole in the $\mathrm{X}^{-}$ground state (top rows), and wave functions of the two lowest electron orbitals with $A^{\prime}$ symmetry (bottom rows), as a function of the edge charge magnitude. The edge charge is located on the top edge, in this view. The strongest $\mathrm{SU}$ peak corresponds to $Q_{e d g e} \approx 0.4$, when the $\mathrm{X}^{-}$ electron charge density reveals a clear contribution from $(2,1,1)_{e}$, and the hole is not yet fully dissociated from electrons.

At $Q_{\text {edge }} \approx 0.4$, symmetry lowering and energetic considerations enable efficient Coulomb coupling. The oval shape of the two-electron charge density reflects a significant contribution from $(2,1,1)_{e}$ to $\left|G S_{X^{-}}\right\rangle$(i.e. $\left.\left|c_{2}\right|>0\right)$. At the same time, the electron $(1,1,1)_{e}$ orbital and the hole ground state have sizable overlap. This is an optimal situation for the appearance for the transition $P_{G S \rightarrow(2,1,1)_{e}}$ to show up as a SU process, according to Equation (3). Further increasing $Q_{\text {edge }}$ separates the $(2,1,1)_{e}$ electron orbital from the hole. Coulomb attraction is then weaker, making $c_{2}$ and consequently $P_{G S \rightarrow(2,1,1)_{e}}$ small again.

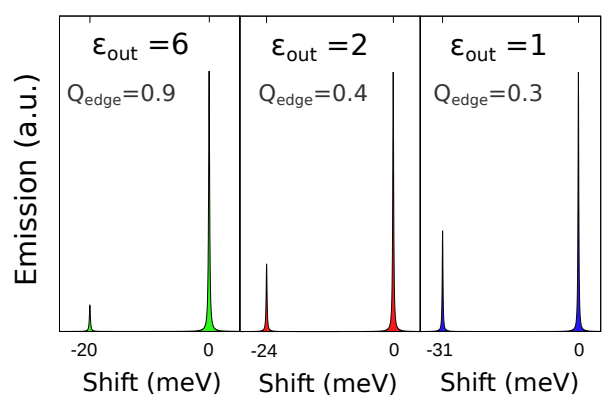

Figure 4: Normalized $\mathrm{X}^{-}$emission as a function of the environment dielectric constant. With increasing dielectric contrast, the SU peak increases and becomes more redshifted. For every value of $\epsilon_{\text {out }}$, the value of $Q_{\text {edge }}$ that maximizes $\mathrm{SU}$ transitions is shown. In all cases, $\epsilon_{\text {in }}=6$.

We have argued above that strong Coulomb admixture of configurations facilitates the appearance of SU processes. A distinct feature of colloidal NPLs when compared to epitaxial quantum wells is the presence of a pronounced dielectric contrast with the organic 
ligands surrounding the NPL, which enhances Coulomb interactions by effectively reducing the system dielectric screening. ${ }^{36,41,43}$ To study the influence of this phenomenon over SU transitions, in Figure 4 we compare the trion emission spectrum for different values of the environment dielectric constant $\epsilon_{\text {out }}$, while fixing that of the NPL to the high-frequency CdSe value, $\epsilon_{i n}=6$. For the sake of comparison, the emission spectrum is normalized so that the band edge peak has the same intensity in all cases. Also, we have selected the value of $Q_{\text {edge }}$ that maximizes the relative size of the $\mathrm{SU}$ peak in each case. Because $\epsilon_{\text {out }}$ screens the surface charge electrostatic field, larger $Q_{\text {edge }}$ values are needed when $\epsilon_{\text {out }}$ increases. The figure evidences that lowering $\epsilon_{\text {out }}$ increases the SU peak height and energetic redshift. For typical ligands of CdSe NPLs (e.g. oleic acid), $\epsilon_{\text {out }} \sim 2 .{ }^{36,44}$ We then conclude that dielectric confinement makes SU processes in colloidal NPLs more conspicuous.

\section{Core/shell NPLs}

We next consider heterostructured core/shell NPLs. The first case under study are CdSe/CdS NPLs. ${ }^{12,13,17,45}$ The NPLs have the same CdSe core as in the previous section and 6 ML thick CdS shells on top and bottom (see inset in Figure 5a). In general, the behavior of SU replicas is found to be analogous to that of core-only NPLs. An off-centered acceptor impurity is needed to yield sizable $\mathrm{SU}$ replicas, with an optimal value of $Q_{\text {edge }}$ maximizing the relative size of the SU peak.

Figure 5a shows the emission spectrum of $\mathrm{X}^{-}$for the optimal $Q_{\text {edge }}$ value, in $\mathrm{CdSe} / \mathrm{CdS}$ NPLs (green line) and CdSe core-only NPLs (black, dashed line). One can see that the SU replica of the CdSe/CdS structure is again significant (11\% of the main transition), but less pronounced than in the core-only structure $(26 \%)$. The smaller SU replica in the core/shell structures is a robust result, which holds for different shell thicknesses and surface charge locations. It is a consequence of the weaker Coulomb interactions. The electron leakage into the CdS shell reduces electron-electron repulsions and electron-hole attractions. The quenching of dielectric confinement by the CdS shell, which pushes organic ligands far from 

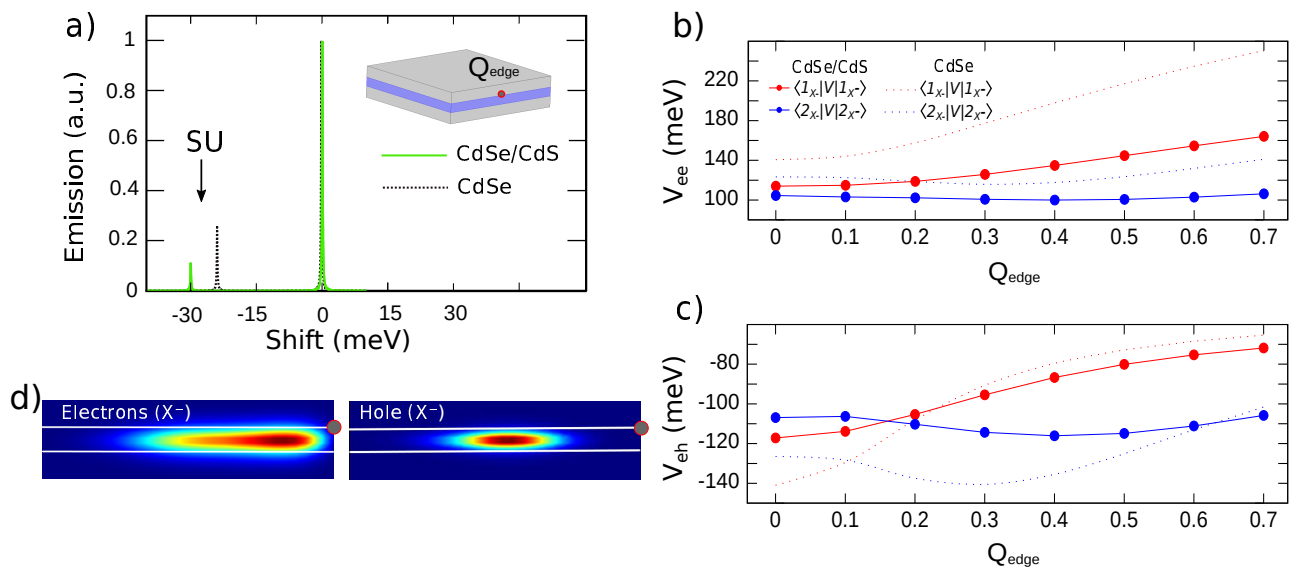

Figure 5: (a) Normalized $\mathrm{X}^{-}$emission spectrum in a CdSe/CdS NPL with 6 ML-thick shell (solid line), compared to that of a core-only CdSe NPL (dotted line). The spectra are centered at the energy of the band edge transition. $Q_{\text {edge }}=0.6(0.4)$ is used for the CdSe/CdS NPL (core-only NPL), to maximize the relative height of SU lines. The SU peak for the core/shell system is smaller than for core-only NPLs. (b,c) Average Coulomb integrals of $\left|G S_{X^{-}}\right\rangle$configurations $\left|1_{X^{-}}\right\rangle$and $\left|2_{X^{-}}\right\rangle$: (b) electron-electron repulsion, (c) electron-hole attraction. Solid (dotted) lines are used for core/shell (core-only) NPLs. The interactions are weaker in the core/shell structure. (d) Charge densities of electrons (left) and hole (right) for the trion ground state in the CdSe/CdS NPL at $Q_{\text {edge }}=0.6$. The electron stays in the vicinity of the core, despite the shallow band offset.

the core, further contributes to the weakening. This observation is reflected in Figs. 5b and $5 \mathrm{c}$, which show that Coulomb interactions (especially $V_{e e}$ ) are weakened in core/shell NPLs (solid lines) as compared to core-only NPLs (dotted lines). Configuration $\left|2_{X^{-}}\right\rangle$is then less stabilized with respect to $\left|1_{X^{-}}\right\rangle$, which implies smaller $\left|c_{2}\right|$ coefficient in the CI expansion.

Figure 5d compares the charge density of the two electrons (left) and hole (right) in $\left|G S_{X^{-}}\right\rangle$. The trion electrons are found to stay in the vicinity of the core, rather than delocalizing all over the structure, to benefit from interaction with the hole. This is consistent with the observed behavior of CdSe/CdS NPLs being similar to that of core-only structures, albeit with weakened Coulomb interactions due to the lessened confinement.

Understanding the conditions which promote SU processes allows us to devise structures where their impact would be maximal. In Fig. 6 we consider a core/shell NPL with the same dimensions as before, but CdSe/CdTe composition. The NPL is chosen to be charged with a positive trion $\left(\mathrm{X}^{+}\right)$, since the heavier mass of holes should favor Coulomb admixture as 


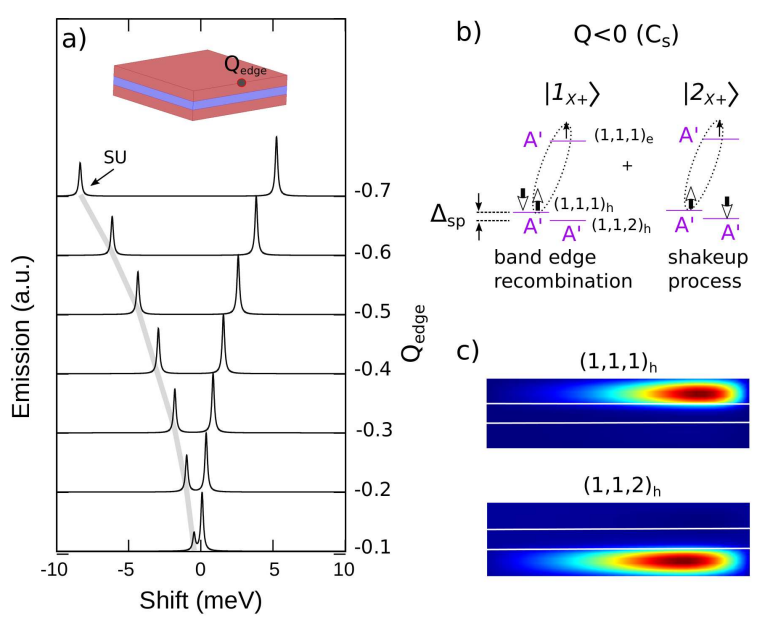

Figure 6: (a) Normalized $\mathrm{X}^{+}$emission spectrum in a CdSe/CdTe NPL with 6 ML thick shells, as a function of the lateral charge strength. The shaded line is a guide to the eye. SU peaks and fundamental transition have comparable intensities. (b) Two main $\left|G S_{X^{+}}\right\rangle$ configurations in the CI expansion in the presence of a charge. The weight of $\left|2_{X^{+}}\right\rangle$is comparable to that of $\left|1_{X^{+}}\right\rangle$in this system, which explains the high SU peaks in (a). (c) Wave function of $(1,1,1)_{h}$ and $(1,1,2)_{h}$ hole orbitals under $Q_{\text {edge }}=-0.5$. The states have the same symmetry but localize on opposite sides of the core to stay orthogonal.

compared to electrons. Because of the type-II band alignment, the electron stays in the CdSe core and the holes in the CdTe region, as observed in related core/crown structures. ${ }^{15,16}$ In the absence of external charges, the two first hole orbitals are $(1,1,1)_{h}$ and $(1,1,2)_{h}$, i.e. the symmetric $\left(A_{1 g}\right)$ and antisymmetric $\left(A_{1 u}\right)$ solutions of the double well potential, respectively, which are almost degenerate because tunneling across the core is negligible (i.e. $\Delta_{s p} \rightarrow 0$ ). Switching on a negative surface charge, $Q_{\text {edge }}<0$, lifts the inversion symmetry so that both orbitals acquire $A^{\prime}$ symmetry and can be Coulomb coupled. The admixture between configurations $\left|1_{X^{+}}\right\rangle$and $\left|2_{X^{+}}\right\rangle$, depicted in Fig. 6b, is then very strong. In the presence of the charge, the two hole orbitals tend to localize on opposite shell sides to remain orthogonal, as shown in Fig. 6c. This implies that configuration $\left|1_{X^{+}}\right\rangle$, which has two holes in the same orbital, has much stronger repulsion than configuration $\left|2_{X^{+}}\right\rangle$, which distributes the two electrons on opposite sides of the core. This makes $\left\langle 1_{X^{+}}\left|V_{h h}\right| 1_{X^{+}}\right\rangle \gg\left\langle 2_{X^{+}}\left|V_{h h}\right| 2_{X^{+}}\right\rangle$. Altogether, the small $\Delta_{s p}$ value and the large difference in hole-hole repulsion explain the strong admixture between configurations $\left|1_{X^{+}}\right\rangle$and $\left|2_{X^{+}}\right\rangle$. As shown in Fig. 6a, this gives 
rise to $\mathrm{SU}$ peaks whose magnitude is almost as large as that of the fundamental transition $\left(72 \%\right.$ for $\left.Q_{\text {edge }}=-0.5\right)$.

It is worth noting that trions in type-II NPLs, having stronger repulsions than attractions, are susceptible of displaying a sizable SU peak even in the absence of trap charges. We noticed this behavior in earlier simulations of rectangular shaped ( $D_{2 h}$ symmetry) core/crown CdSe/CdTe NPLs. ${ }^{32}$

\section{Discussion}

Our simulations show that SU processes can be expected for trions in core-only and core/shell NPLs, if off-centered charges are present. We discuss here the potential relationship of this finding with experiments in the literature and practical implications.

\section{Relationship with experiments}

In core-only CdSe NPLs, the low temperature photoluminescence is thought to arise from subpopulations of excitons and negative trions. ${ }^{24,33,46}$ Very recently, Antolinez and co-workers have reported that the $\mathrm{X}^{-}$emission shows a distinct peak or a shoulder (depending on the film thickness) redshifted from the trion band edge transition. The redshift is $\sim 19 \mathrm{meV}$ and the relative height $15-25 \%$ that of the main peak. ${ }^{24}$ They speculated that the origin could be a SU process of the kind we study. Our calculations support the feasibility of this interpretation. Figure 1a shows excellent agreement with the experimental measurements, both in energy and relative intensity of the SU peak, assuming a lateral charge with $Q_{\text {edge }}=$ $0.3-0.4$, which gives a redshift of $19-25 \mathrm{meV}$ and a relative height of $15-23 \%$.

The presence of acceptor charges in CdSe NPLs likely originates when the hole of a photoexcited electron-hole pair is trapped by a surface defect. The next electron-hole pair generated in the NPL joins the residual electron to form $\mathrm{X}^{-}$, while the trapped hole exerts a screened electrostatic potential. ${ }^{22,38,47}$ The coexistence of $\mathrm{X}^{-}$and trapped surface charges in 
CdSe NPLs is backed up by studies reporting correlation between surface-to-volume ratio, laser irradiation time and trion emission intensity. ${ }^{46}$ A plausible location for surface charges are the lateral sidewalls of the NPL (as in Fig. 1b). This possibility is suggested by studies showing that Z-type ligand desorption -and hence surface traps- in CdSe NPLs is more frequent on these facets, ${ }^{48}$ and by the fact that CdSe/CdS core/crown NPLs generally improve the photoluminescence quantum yield as compared to core-only structures, despite having larger surfaces on top and bottom. ${ }^{14}$ Because off-centered charges are needed to originate SU peaks, lateral charges are candidates to trigger such processes.

In core/shell CdSe/CdS NPLs, SU processes have been also proposed as the origin of multi-peaked fluorescence emission -and hence broadened line width- ${ }^{23}$ Our simulations in Fig. 5a confirm one can indeed expect a sizable SU peak in such structures. We note that earlier experimental studies had so far interpreted the line width broadening as a result of either SU processes ${ }^{23}$ or of surface defects. ${ }^{12}$ By showing that the second effect is a prerequesite for the first one, our study helps to reconcile both interpretations. Nonetheless, two remarkable disagreements are observed between our simulations and Ref. ${ }^{23}$ measurements. First, the experiments show from 2 to 4 emission peaks, which are interpreted as the $\mathrm{X}^{-}$ fundamental transition plus up to three redshifted, SU peaks. In our calculations, however, we fail to see more than one significant SU replica. Second, the highest-energy peak in the experiment is never the brightest one. This is inconsistent with our results and with earlier studies on epitaxial quantum wells and dots, where the higher-energy peak corresponds to the fundamental transition, which is the most likely recombination channel. ${ }^{25-29}$

Tentatively, one may suspect that a large number of SU peaks in core/shell CdSe/CdS NPLs could be connected with the thick CdS shell (12 ML in Ref. ${ }^{23}$ ), which makes surface defects more likely than in core-only structures. A significant presence of defects in these structures has been hinted by studies showing that the long radiative lifetime is not due to electron delocalization but to the influence of impurities. ${ }^{13}$ However, Coulomb interactions are weaker than in core-only structures (Fig. 5c,d), where only one SU peak has been mea- 
sured. ${ }^{24}$ It is then not surprising that, despite investigating different charge locations (Figs. S3, S6 and S7 in SI), conduction band-offset values (Fig. S4) and shell thicknesses (Fig.S5), we see at most one significant SU satellite. Deviations from the squared core shape, despite lowering the symmetry, do not change this result either (see Fig.S8).

Regarding the relative intensity of the peaks, as mentioned in the previous section, the highest-energy one (fundamental transition) is proportional to the weight of configuration $\left|1_{X^{-}}\right\rangle$in the CI expansion, $\left|c_{1}\right|^{2}$, while subsequent (SU) peaks would be proportional to $\left|c_{2}\right|^{2},\left|c_{3}\right|^{2}, \ldots$ Configuration $\left|1_{X^{-}}\right\rangle$(all carriers in the ground orbital, Fig. 2c) is nodeless and hence naturally expected to be the dominant one, so the highest-energy peak is also the brightest one. We have not observed SU peaks exceeding the fundamental transition height despite considering different charge locations and shell thicknesses (see SI). Even in CdSe/CdTe NPLs, which constitute a limit case, SU peaks never exceed the height of the main transition, see Fig. 6a.
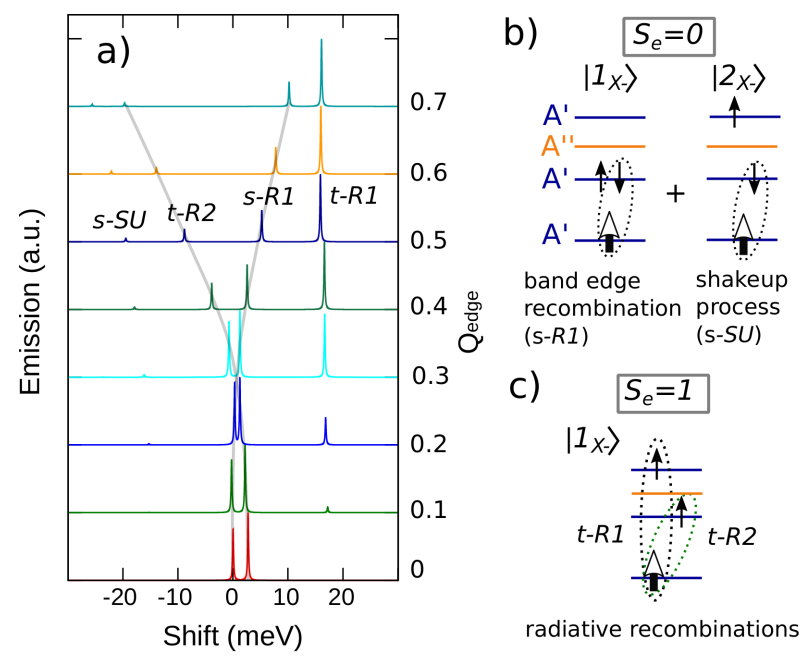

Figure 7: (a) Normalized $\mathrm{X}^{-}$emission spectrum in a CdSe/CdS NPL, as a function of the lateral charge strength. An electron spin relaxation bottleneck is imposed, so that emission comes from the lowest singlet $\left(S_{e}=0\right.$, ground state) and triplet $\left(S_{e}=1\right)$ states. Shaded lines are guides to the eyes. (b-c) Sketches showing the relevant electron-hole recombination channels of singlet and triplet states. (b) The singlet can give rise to one fully radiative $(s-R 1)$ plus one SU transition $(s-S U)$. (c) The triplet can give rise to two fully radiative transitions, $t-R 1$ and $t-R 2$.

We conclude from our simulations that SU lines may be present in the spectra of CdSe/CdS 
NPLs, but they are unlikely to explain all the features observed in Ref. ${ }^{23}$ As an alternative interpretation for the experiments, a multi-peaked emission spectrum could result from stacking of colloidal NPLs, ${ }^{49}$ which leads to electronic coupling through dielectric confinement. ${ }^{50}$ However, the time-dependent spectral shifts observed by Antolinez et al. suggest that all peaks arise from the same NPL, and significant stacking was not expected in the experiment samples. ${ }^{23}$ We thus propose a different interpretation. Namely, simultaneous emission from the $\mathrm{X}^{-}$ground state, with singlet electron spin $\left(S_{e}=0\right)$, and a metastable excited state with triplet electron spin $\left(S_{e}=1\right)$. The decay from the triplet to the singlet state is slowed down by spin selection rules, as phonons are spinless. This could allow simultaneous occupation of the two states even if the energy splitting exceeds thermal energy.

To illustrate this point, in Figure $7 \mathrm{a}$ we show the calculated emission of $\mathrm{X}^{-}$assuming equipopulation of $S_{e}=0$ and $S_{e}=1$ trion states. One can see that the number of sizable peaks in the spectrum ranges from two to four, depending on the strength of surface charge, $Q_{\text {edge. }}$ The origin of these peaks is summarized in the sketches of Fig. 7b and 7c. The singlet (Fig. 7b) can give rise to a fully radiative transition $(s-R 1)$ and a $\mathrm{SU}$ transition $(s-S U)$, as described in the previous sections. In turn, the triplet (Fig. 7c) can give rise to two fully radiative transitions ( $t-R 1$ and $t-R 2)$, depending on which electron recombines with the hole. $t$ - $R 2$ is readily visible at $Q_{\text {edge }}=0$, but $t$ - $R 1$ requires recombining the hole with an excited electron, a process which is again activated when the surface charge lifts symmetry restrictions. However, unlike in SU processes, the two triplet transitions come from the main configuration of the trion CI expansion. Therefore, their intensity can be comparable to that of the band edge transition, $s-S U$, even if Coulomb admixture is weak. The triplet transitions are built on both sides of $s-S U$, with inter-peak energy splittings up to few tens of meV. The relative sizes of the peaks will be further modulated in realistic situations by a finite triplet-singlet decay rate. This relaxation channel would possibly reduce the relative population of $S_{e}=1$, and hence the intensity of $t$ - $R 1$.

Altogether, the number of peaks, the magnitude of the energy splitting between the peaks 
and the flexible intensities provide a framework to explain the multi-peaked photoluminescence of Ref. ${ }^{23}$ Several other aspects of this proposal are consistent with the experiments. For example, because all peaks in Fig. 7a arise from the same NPL, they will experience simultaneous spectral shifts when surface impurities migrate. ${ }^{23}$ Also, the hot trion emission is expected to vanish when the impurities are removed, as $t$ - $R 1$ becomes deactivated and $t$ - $R 1$ almost merges with the singlet emission, $s-R 1$, see Fig. 7a for $Q_{e d g e}=0$. This agrees with the experimental observation that the line shape evolves from asymmetric to symmetric as temperature increases. ${ }^{12}$

The fact that triplet emission is observed in CdSe/CdS NPLs, but not in CdSe ones, may be explained from the strong spin-spin interaction of resident carriers and surface dangling bonds in the latter case, ${ }^{51}$ which should speed up spin relaxation through flip/flop processes. This mechanism is expected to be inhibited in core/shell structures, because $\mathrm{X}^{-}$carriers stay far from the surface, as shown in Fig. 5d. On the other hand, the triplet trion is expected to have fine structure through electron-hole exchange interaction, ${ }^{52}$ which may not fit the mono-exponential photoluminescence decay reported in Ref. ${ }^{23}$ Further experiments are needed, e.g. on polarisation of the different peaks under external fields ${ }^{33,53}$ or magnetic dispersion ${ }^{31}$ to confirm the different spin of the emissive states in CdSe/CdS NPLs.

The observation of metastable triplet trion photoluminescence has been previously reported in epitaxial quantum wells ${ }^{27,54}$ and dots, ${ }^{53}$ and more recently in transition metal chalcogenide monolayers. ${ }^{55}$ To our knowledge, however, its presence in colloidal nanostructures has not been confirmed.

\section{Control of SU processes}

Inasmuch as SU processes can be responsible for the line width broadening NPLs, their supression is desirable to improve color purity in optical applications. It has been suggested that this job could be achieved by increasing quantum confinement, reducing either lateral dimensions or shell thickness - the latter would favor electrostatic confinement. ${ }^{23}$ Both 
strategies have the drawback of introducing size dispersion in ensemble luminescence. From our theoretical analysis, we confirm that reducing Coulomb admixture would minimize SU processes, but this can be achieved by weakening Coulomb interactions instead of increasing quantum confinement. For example, reducing dielectric confinement or using thinner cores to enhance the quasi-type-II character should contribute to this goal. Obviously, this approach would have the drawback of reducing the band edge recombination rate as well.

Alternatively, since our study shows that trap charges are ultimately responsible for SU processes, experimental routes to suppress SU processes could be directed to control of traps. Appropriate choice of surface ligands, ${ }^{48}$ electrochemical potentials ${ }^{56}$ and interface alloying ${ }^{19,20}$ could contribute to this end.

Because we find surface charges on lateral sidewalls particularly suited to induce SU processes, the growth of core/crown heterostructures is expected to reduce their influence by keeping the outer rim away from the photogenerated carriers. This suggestion seems to agree with experimental observations by Kelestemur and co-workers, indicating that core/crown/shell CdSe/CdS NPLs have more symmetric emission behavior than core/shell ones at cryogenic temperatures ${ }^{57}$ This can be understood as a consequence of the suppression of SU processes in the low-energy tail of the emission band. It is also consistent with recent single-particle studies showing that the line width in CdTe/CdSe core/crown NPLs is set by LO phonon replica of neutral excitons, rather than SU peaks of trions. ${ }^{32}$

Should the role of metastable triplet states be confirmed in CdSe/CdS NPLs, strategies to control the line width should rather focus on enhancing the interaction of confined carriers with surface spins ${ }^{51}$ or intrinsic spin-orbit interaction, ${ }^{58}$ to shorten their lifetime. Replacing trion by neutral exciton emission through thermal dissociation, ${ }^{59}$ is yet another possibility to avoid SU and high spin peaks.

Thus, our calculations propose a wealth of experiments targeted at material design to tune quantum and dielectric confinement, and exciton-surface/interface interactions, and set suitable temperature ranges to control SU/triplet emission. 


\section{Conclusions}

We have shown that SU processes in colloidal NPLs are enabled by severe Coulomb admixture -which results from strong Coulomb interactions and weak lateral confinement- and the presence of off-centered electrostatic traps, which suppress the protection against Auger processes provided by symmetry conservation. Surface charges on lateral sidewalls seem particularly efficient to this end.

Under typical experimental conditions, core-only and core/shell NPLs are susceptible of showing a SU peak with oscillator strength 0.1-0.3 times that of the band edge transition. This is at least one order magnitude larger than in epitaxial quantum wells. The SU peak is redshifted from the band edge peak by up to few tens of $\mathrm{meV}$, thus providing a source of line width broadening.

These results are in excellent agreement with recent experimental findings in CdSe $\mathrm{NPLs}^{24}$ in terms of number of emission peaks, energy splitting and relative intensity, but only partially so with those of core/shell CdSe/CdS NPLs. ${ }^{23}$ Experiments in the latter structure are however in line with an alternative interpretation involving simultaneous participation from trion singlet and metastable triplet states.

Strategies to narrow the line width of NPLs through suppression of SU processes should aim at controlling charge traps or Coulomb admixture.

\section{Methods}

Calculations are carried within k·p-continuum elastic theory framework. Independent electron and hole states are calculated with single-band Hamiltonians including core/shell strain and self-energy potential terms. Model details and material parameters are given in Ref. ${ }^{45}$ Ligand induced strain ${ }^{60,61}$ cannot be quantified with our continuum model, but its effect on the line width broadening is expected to arise from incomplete passivation ${ }^{60}$ resulting in surface traps. We include such traps as point charges. Point charge electrostatic potentials 
and Coulomb integrals for CI matrix elements, including dielectric mismatch effects, are calculated solving Poisson Equation with Comsol 4.2. The CI basis set is formed by all possible combinations of the first 22 single-electron and 22 single-hole spin-orbitals. For $\mathrm{X}^{-}$, these are combined to form configurations $\left|m_{X^{-}}\right\rangle$as the Hatree product of one hole spin-orbital with a two-electron Slater determinant.

\section{Supporting Information}

This material is available free of charge via the internet at http://pubs.acs.org.

Additional calculations on the influence of the trion sign, surface charge location, conduction band offset and NPL core shape over the formation of SU processes.

\section{Acknowledgement}

The authors acknowledge support from MICINN project CTQ2017-83781-P. We are grateful to I. Moreels, A. Achtstein and F. Rabouw for useful discussions.

\section{References}

(1) Lhuillier, E.; Pedetti, S.; Ithurria, S.; Nadal, B.; Heuclin, H.; Dubertret, B. Twodimensional colloidal metal chalcogenides semiconductors: synthesis, spectroscopy, and applications. Accounts of Chemical Research 2015, 48, 22-30.

(2) Min, Y.; Im, E.; Hwang, G.-T.; Kim, J.-W.; Ahn, C.-W.; Choi, J.-J.; Hahn, B.-D.; Choi, J.-H.; Yoon, W.-H.; Park, D.-S., et al. Heterostructures in two-dimensional colloidal metal chalcogenides: Synthetic fundamentals and applications. Nano Research 2019, 1-20.

(3) Diroll, B. T. Colloidal quantum wells for optoelectronic devices. Journal of Materials Chemistry C 2020, 8, 10628-10640. 
(4) Sharma, M.; Delikanli, S.; Demir, H. V. Two-Dimensional CdSe-Based Nanoplatelets: Their Heterostructures, Doping, Photophysical Properties, and Applications. Proceedings of the IEEE 2019, 108, 655-675.

(5) Feldmann, J.; Peter, G.; Göbel, E.; Dawson, P.; Moore, K.; Foxon, C.; Elliott, R. Linewidth dependence of radiative exciton lifetimes in quantum wells. Physical Review Letters 1987, 59, 2337.

(6) Planelles, J.; Achtstein, A. W.; Scott, R.; Owschimikow, N.; Woggon, U.; Climente, J. I. Tuning intraband and interband transition rates via excitonic correlation in lowdimensional semiconductors. ACS Photonics 2018, 5, 3680-3688.

(7) Ithurria, S.; Tessier, M.; Mahler, B.; Lobo, R.; Dubertret, B.; Efros, A. L. Colloidal nanoplatelets with two-dimensional electronic structure. Nature Materials 2011, 10, $936-941$.

(8) Riedinger, A.; Ott, F. D.; Mule, A.; Mazzotti, S.; Knüsel, P. N.; Kress, S. J.; Prins, F.; Erwin, S. C.; Norris, D. J. An intrinsic growth instability in isotropic materials leads to quasi-two-dimensional nanoplatelets. Nature Materials 2017, 16, 743-748.

(9) Christodoulou, S.; Climente, J. I.; Planelles, J.; Brescia, R.; Prato, M.; MartínGarcía, B.; Khan, A. H.; Moreels, I. Chloride-induced thickness control in CdSe nanoplatelets. Nano letters 2018, 18, 6248-6254.

(10) Bhandari, G. B.; Subedi, K.; He, Y.; Jiang, Z.; Leopold, M.; Reilly, N.; Lu, H. P.; Zayak, A. T.; Sun, L. Thickness-controlled synthesis of colloidal PbS nanosheets and their thickness-dependent energy gaps. Chemistry of Materials 2014, 26, 5433-5436.

(11) Tessier, M. D.; Javaux, C.; Maksimovic, I.; Loriette, V.; Dubertret, B. Spectroscopy of single CdSe nanoplatelets. ACS nano 2012, 6, 6751-6758. 
(12) Tessier, M.; Mahler, B.; Nadal, B.; Heuclin, H.; Pedetti, S.; Dubertret, B. Spectroscopy of colloidal semiconductor core/shell nanoplatelets with high quantum yield. Nano letters 2013, 13, 3321-3328.

(13) Achtstein, A. W.; Marquardt, O.; Scott, R.; Ibrahim, M.; Riedl, T.; Prudnikau, A. V.; Antanovich, A.; Owschimikow, N.; Lindner, J. K.; Artemyev, M., et al. Impact of Shell Growth on Recombination Dynamics and Exciton-Phonon Interaction in CdSe-CdS Core-Shell Nanoplatelets. ACS nano 2018, 12, 9476-9483.

(14) Tessier, M. D.; Spinicelli, P.; Dupont, D.; Patriarche, G.; Ithurria, S.; Dubertret, B. Efficient exciton concentrators built from colloidal core/crown CdSe/CdS semiconductor nanoplatelets. Nano letters 2014, 14, 207-213.

(15) Antanovich, A.; Prudnikau, A.; Melnikau, D.; Rakovich, Y. P.; Chuvilin, A.; Woggon, U.; Achtstein, A. W.; Artemyev, M. Colloidal synthesis and optical properties of type-II CdSe-CdTe and inverted CdTe-CdSe core-wing heteronanoplatelets. Nanoscale 2015, 7, 8084-8092.

(16) Kelestemur, Y.; Olutas, M.; Delikanli, S.; Guzelturk, B.; Akgul, M. Z.; Demir, H. V. Type-II colloidal quantum wells: $\mathrm{CdSe} / \mathrm{CdTe}$ core/crown heteronanoplatelets. The Journal of Physical Chemistry C 2015, 119, 2177-2185.

(17) Polovitsyn, A.; Dang, Z.; Movilla, J. L.; Martín-García, B.; Khan, A. H.; Bertrand, G. H.; Brescia, R.; Moreels, I. Synthesis of air-stable CdSe/ZnS core-shell nanoplatelets with tunable emission wavelength. Chem. Mater. 2017, 29, 5671-5680.

(18) Saidzhonov, B.; Kozlovsky, V.; Zaytsev, V.; Vasiliev, R. Ultrathin CdSe/CdS and CdSe/ZnS core-shell nanoplatelets: The impact of the shell material on the structure and optical properties. Journal of Luminescence 2019, 209, 170-178.

(19) Rossinelli, A. A.; Rojo, H.; Mule, A. S.; Aellen, M.; Cocina, A.; De Leo, E.; Schäublin, R.; Norris, D. J. Compositional Grading for Efficient and Narrowband Emis- 
sion in CdSe-Based Core/Shell Nanoplatelets. Chemistry of Materials 2019, 31, 95679578.

(20) Kelestemur, Y.; Shynkarenko, Y.; Anni, M.; Yakunin, S.; De Giorgi, M. L.; Kovalenko, M. V. Colloidal CdSe Quantum Wells with Graded Shell Composition for Low-Threshold Amplified Spontaneous Emission and Highly Efficient Electroluminescence. ACS nano 2019, 13, 13899-13909.

(21) Yadav, S.; Singh, A.; Thulasidharan, L.; Sapra, S. Surface decides the photoluminescence of colloidal CdSe nanoplatelets based core/shell heterostructures. The Journal of Physical Chemistry C 2018, 122, 820-829.

(22) Rabouw, F. T.; van der Bok, J. C.; Spinicelli, P.; Mahler, B.; Nasilowski, M.; Pedetti, S.; Dubertret, B.; Vanmaekelbergh, D. Temporary charge carrier separation dominates the photoluminescence decay dynamics of colloidal CdSe nanoplatelets. Nano Letters $\mathbf{2 0 1 6}$ $16,2047-2053$.

(23) Antolinez, F. V.; Rabouw, F. T.; Rossinelli, A. A.; Cui, J.; Norris, D. J. Observation of Electron Shakeup in CdSe/CdS Core/Shell Nanoplatelets. Nano Lett. 2019, 19, 84958502.

(24) Antolinez, F. V.; Rabouw, F. T.; Rossinelli, A. A.; Keitel, R. C.; Cocina, A.; Becker, M. A.; Norris, D. J. Trion Emission Dominates the Low-Temperature Photoluminescence of CdSe Nanoplatelets. Nano Letters 2020, 20, 5814-5820.

(25) Nash, K.; Skolnick, M.; Saker, M.; Bass, S. Many body shakeup in quantum well luminescence spectra. Physical Review Letters 1993, 70, 3115.

(26) Finkelstein, G.; Shtrikman, H.; Bar-Joseph, I. Shakeup processes in the recombination spectra of negatively charged excitons. Physical Review B 1996, 53, 12593. 
(27) Bryja, L.; Wójs, A.; Misiewicz, J.; Potemski, M.; Reuter, D.; Wieck, A. Magneto-optical probing of weak disorder in a two-dimensional hole gas. Physical Review B 2007, 75, 035308 .

(28) Dzyubenko, A. Shake-up processes in a low-density two-dimensional electron gas: Spindependent transitions to higher hole Landau levels. Physical Review B 2004, 69, 115332.

(29) Paskov, P.; Holtz, P.-O.; Wongmanerod, S.; Monemar, B.; Garcia, J.; Schoenfeld, W.; Petroff, P. M. Auger processes in InAs self-assembled quantum dots. Physica E: Lowdimensional Systems and Nanostructures 2000, 6, 440-443.

(30) Hawrylak, P. Resonant magnetoexcitons and the fermi-edge singularity in a magnetic field. Physical Review B 1991, 44, 11236.

(31) Löbl, M. C.; Spinnler, C.; Javadi, A.; Zhai, L.; Nguyen, G. N.; Ritzmann, J.; Midolo, L.; Lodahl, P.; Wieck, A. D.; Ludwig, A., et al. Radiative Auger process in the singlephoton limit. Nature Nanotechnology 2020, 15, 558-562.

(32) Steinmetz, V. et al. Emission State Structure and Linewidth Broadening Mechanisms in Type-II CdSe/CdTe Core-Crown Nanoplatelets: A Combined Theoretical - Single Nanocrystal Optical Study. The Journal of Physical Chemistry C 2020, 124, 1735217363.

(33) Shornikova, E. V.; Yakovlev, D. R.; Biadala, L.; Crooker, S. A.; Belykh, V. V.; Kochiev, M. V.; Kuntzmann, A.; Nasilowski, M.; Dubertret, B.; Bayer, M. Negatively charged excitons in CdSe nanoplatelets. Nano Letters 2020, 20, 1370-1377.

(34) Shornikova, E. V.; Biadala, L.; Yakovlev, D. R.; Feng, D.; Sapega, V. F.; Flipo, N.; Golovatenko, A. A.; Semina, M. A.; Rodina, A. V.; Mitioglu, A. A., et al. Electron and hole g-factors and spin dynamics of negatively charged excitons in CdSe/CdS colloidal nanoplatelets with thick shells. Nano letters 2018, 18, 373-380. 
(35) Adachi, S. Handbook on Physical Properties of Semiconductors, vol.3; Kluwer Academics, 2004.

(36) Achtstein, A. W.; Schliwa, A.; Prudnikau, A.; Hardzei, M.; Artemyev, M. V.; Thomsen, C.; Woggon, U. Electronic structure and exciton-phonon interaction in twodimensional colloidal CdSe nanosheets. Nano Lett. 2012, 12, 3151-3157.

(37) Beyler, A. P.; Marshall, L. F.; Cui, J.; Brokmann, X.; Bawendi, M. G. Direct observation of rapid discrete spectral dynamics in single colloidal CdSe-CdS core-shell quantum dots. Physical Review Letters 2013, 111, 177401.

(38) Califano, M.; Franceschetti, A.; Zunger, A. Temperature Dependence of Excitonic Radiative Decay in CdSe Quantum Dots: The Role of Surface Hole Traps. Nano Letters 2005, 5, 2360-2364.

(39) Drijvers, E.; De Roo, J.; Martins, J. C.; Infante, I.; Hens, Z. Ligand displacement exposes binding site heterogeneity on CdSe nanocrystal surfaces. Chemistry of Materials 2018, 30, 1178-1186.

(40) Jacak, L.; Hawrylak, P.; Wojs, A. Quantum Dots; Springer, 1998.

(41) Rajadell, F.; Climente, J. I.; Planelles, J. Excitons in core-only, core-shell and corecrown CdSe nanoplatelets: Interplay between in-plane electron-hole correlation, spatial confinement, and dielectric confinement. Phys. Rev. B 2017, 96, 035307.

(42) Richter, M. Nanoplatelets as material system between strong confinement and weak confinement. Phys. Rev. Mater. 2017, 1, 016001.

(43) Benchamekh, R.; Gippius, N. A.; J., E.; Nestoklon, M. O.; Jancu, J.-M.; Ithurria, S.; Dubertret, B.; Efros, A. L.; P., V. Tight-Binding Calculations of Image-Charge Effects in Colloidal Nanoscale Platelets of CdSe. Phys. Rev. B: Condens. Matter Mater. Phys. 2014, 89, 035307. 
(44) Even, J.; Pedesseau, L.; Kepenekian, M. Electronic surface states and dielectric selfenergy profiles in colloidal nanoscale platelets of CdSe. Physical Chemistry Chemical Physics 2014, 16, 25182-25190.

(45) Llusar, J.; Planelles, J.; Climente, J. I. Strain in Lattice-Mismatched CdSe-Based Core/Shell Nanoplatelets. The Journal of Physical Chemistry C 2019, 123, 2129921306.

(46) Yu, J.; Zhang, C.; Pang, G.; Sun, X. W.; Chen, R. Effect of Lateral Size and Surface Passivation on the Near-Band-Edge Excitonic Emission from Quasi-Two-Dimensional CdSe Nanoplatelets. ACS applied materials \& interfaces 2019, 11, 41821-41827.

(47) Feng, D.; Yakovlev, D. R.; Pavlov, V. V.; Rodina, A. V.; Shornikova, E. V.; Mund, J.; Bayer, M. Dynamic evolution from negative to positive photocharging in colloidal CdS quantum dots. Nano Letters 2017, 17, 2844-2851.

(48) Leemans, J.; Singh, S.; Li, C.; Ten Brinck, S.; Bals, S.; Infante, I.; Moreels, I.; Hens, Z. Near-Edge Ligand Stripping and Robust Radiative Exciton Recombination in CdSe/CdS Core/Crown Nanoplatelets. The Journal of Physical Chemistry Letters 2020, 11, 3339-3344.

(49) Diroll, B. T.; Cho, W.; Coropceanu, I.; Harvey, S. M.; Brumberg, A.; Holtgrewe, N.; Crooker, S. A.; Wasielewski, M. R.; Prakapenka, V. B.; Talapin, D. V., et al. Semiconductor nanoplatelet excimers. Nano letters 2018, 18, 6948-6953.

(50) Movilla, J. L.; Planelles, J.; Climente, J. I. Dielectric Confinement Enables Molecular Coupling in Stacked Colloidal Nanoplatelets. J. Phys. Chem. Lett. 2020, 11, 3294-3300.

(51) Shornikova, E. V.; Golovatenko, A. A.; Yakovlev, D. R.; Rodina, A. V.; Biadala, L.; Qiang, G.; Kuntzmann, A.; Nasilowski, M.; Dubertret, B.; Polovitsyn, A., et al. Surface spin magnetism controls the polarized exciton emission from CdSe nanoplatelets. Nature Nanotechnology 2020, 15, 277-282. 
(52) Ware, M.; Stinaff, E.; Gammon, D.; Doty, M.; Bracker, A.; Gershoni, D.; Korenev, V.; Bădescu, Ş.; Lyanda-Geller, Y.; Reinecke, T. Polarized fine structure in the photoluminescence excitation spectrum of a negatively charged quantum dot. Physical Review Letters 2005, 95, 177403.

(53) Jovanov, V.; Kapfinger, S.; Bichler, M.; Abstreiter, G.; Finley, J. Direct observation of metastable hot trions in an individual quantum dot. Physical Review B 2011, 84, 235321.

(54) Shields, A.; Osborne, J.; Simmons, M.; Pepper, M.; Ritchie, D. Magneto-optical spectroscopy of positively charged excitons in GaAs quantum wells. Physical Review B 1995, 52, R5523.

(55) Vaclavkova, D.; Wyzula, J.; Nogajewski, K.; Bartos, M.; Slobodeniuk, A.; Faugeras, C.; Potemski, M.; Molas, M. Singlet and triplet trions in WS2 monolayer encapsulated in hexagonal boron nitride. Nanotechnology 2018, 29, 325705.

(56) Galland, C.; Ghosh, Y.; Steinbrück, A.; Sykora, M.; Hollingsworth, J. A.; Klimov, V. I.; Htoon, H. Two types of luminescence blinking revealed by spectroelectrochemistry of single quantum dots. Nature 2011, 479, 203-207.

(57) Kelestemur, Y.; Guzelturk, B.; Erdem, O.; Olutas, M.; Gungor, K.; Demir, H. V. Platelet-in-Box Colloidal Quantum Wells: CdSe/CdS@ CdS Core/Crown@ Shell Heteronanoplatelets. Advanced Functional Materials 2016, 26, 3570-3579.

(58) Tadjine, A.; Niquet, Y.-M.; Delerue, C. Universal behavior of electron g-factors in semiconductor nanostructures. Physical Review B 2017, 95, 235437.

(59) Ayari, S.; Quick, M. T.; Owschimikow, N.; Christodoulou, S.; Bertrand, G. H.; Artemyev, M.; Moreels, I.; Woggon, U.; Jaziri, S.; Achtstein, A. W. Tuning trion binding energy and oscillator strength in a laterally finite 2D system: CdSe nanoplatelets as a model system for trion properties. Nanoscale 2020, 12, 14448. 
(60) Antanovich, A.; Achtstein, A.; Matsukovich, A.; Prudnikau, A.; Bhaskar, P.; Gurin, V.; Molinari, M.; Artemyev, M. A strain-induced exciton transition energy shift in CdSe nanoplatelets: the impact of an organic ligand shell. Nanoscale 2017, 9, 18042-18053.

(61) Diroll, B. T. Ligand-Dependent Tuning of Interband and Intersubband Transitions of Colloidal CdSe Nanoplatelets. Chemistry of Materials 2020, 32, 5916-5923. 


\section{For Table of Contents Use Only}

Table of contents for manuscript "Nature and Control of Shakeup Processes in Colloidal Nanoplatelets", by Jordi Llusar and Juan I. Climente.

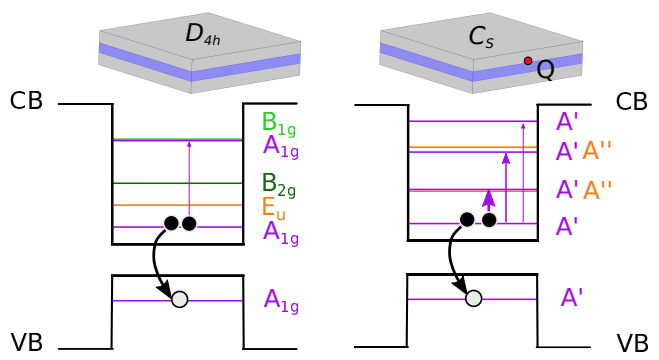

Schematic of radiative Auger (shakeup) process in nanoplatelets (NPLs). Point symmetry largely protects the NPL from such process (left picture), but the presence of charge impurities lowers the symmetry and hence activates shakeup processes (right picture). 


\title{
Supporting Information for “Nature and Control of Shakeup Processes in Colloidal Nanoplatelets"
}

\author{
Jordi Llusar and Juan I. Climente* \\ Departament de Química Física i Analítica, Universitat Jaume I, E-12080, Castelló de la \\ Plana, Spain \\ E-mail: climente@uji.es
}

Abstract

12 pages including 8 figures. 


\section{Additional calculations}

We present here additional calculations for further understanding of SU processes.

\section{Convergence of CI calculations}

Configuration Interaction (CI) calculations on the basis of independent particle (or HartreeFock) orbitals provide an excellent description of repulsions in few- and many-fermion systems. ${ }^{1,2}$ However, large basis sets are needed to describe strong attractions, ${ }^{3,4}$ which are certainly present in colloidal $\mathrm{NPLs}^{5}$ and are involved in a correct description of SU processes.

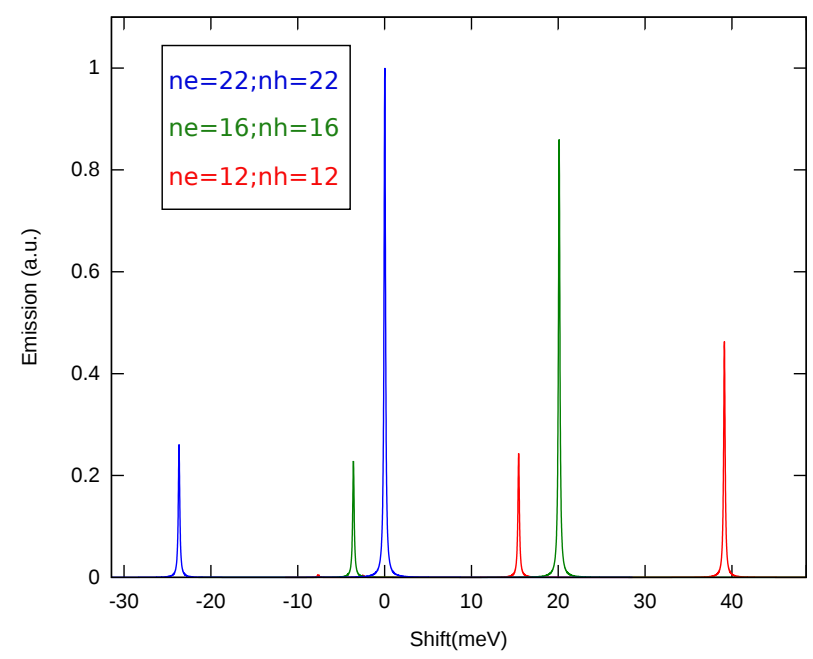

Figure S1: $\mathrm{X}^{-}$emission spectrum for $Q_{\text {edge }}=0.4$ (see main text). Zero energy is set for the fundamental transition with $n e=n h=22$. ne and $n h$ are the number of single-electron and single-hole spin-orbitals, respectively, used to build the CI basis sets.

In Fig. S1 we compare the $\mathrm{X}^{-}$emission spectrum calculated for CdSe NPLs -same dimensions as in main text- using different basis sizes. The basis is formed by all possible combinations of the first ne $(n h)$ independent particle spin-orbital states of electrons (holes). With increasing basis dimensions, the band edge transition peak red-shifts and gains intensity, which reveals an improved description of electron-hole correlation. The intensity of the SU peak height and its red-shift with respect to the band edge transition are however less sensitive to the basis dimensions. It follows from the figure that quantitative assessment on 
the ratio of fundamental vs SU peak heights requires large basis sets. In the main text we use $n e=n h=22$. By comparing with smaller values of $n e / n h$ in the figure, it is clear that for this value -which involves very time-consuming computations- the ratio is reaching saturation. This validates the order of ratios provided in the main text. For the calculations in this Supporting Information, however, we may resort to $n e=n h=12$, which overestimates the relative height of SU peaks, but suffices to provide qualitative assessment.

\section{Positive trion behaviour}

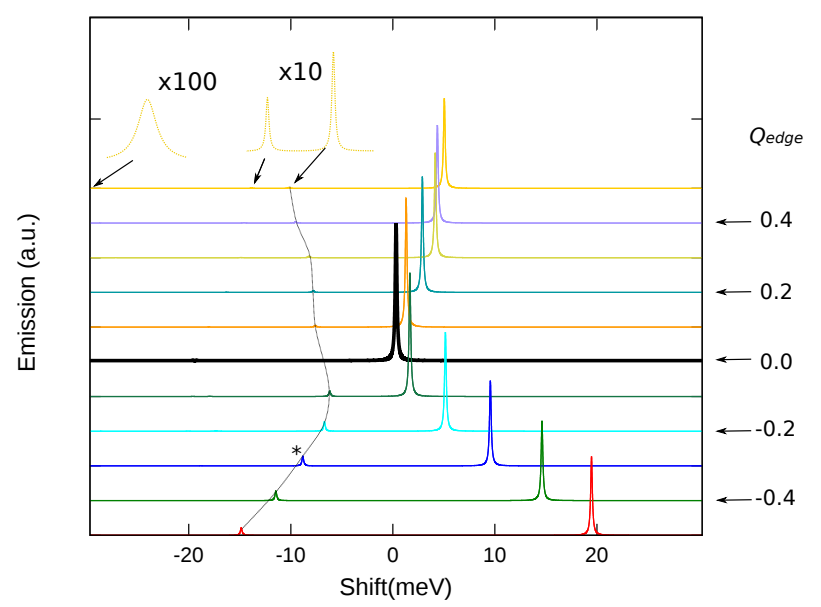

Figure S2: $X^{+}$normalized spectrum emission for different charge intensities. The arrows are pointing to SU satellites (dotted lines are guides to the eyes). The highest SU peak $\left(Q_{\text {edge }}=-0.3\right)$ is marked with a star. The origin of energies is set at the band edge recombination peak. The insets correspond to $Q_{\text {edge }}=0.5$ amplified SU peaks.

In the main text, we have mostly considered the case of negative trions. We show here that the same behavior holds for positive ones. To illustrate this point, we choose the case of the core-only NPL with an edge charge, equivalent to Fig.1d of the main text. Figure S2 shows that the presence of SU peaks in the emission spectrum is again strongly dependent on the value of the surface charge. For $Q_{e d g e}=0$, no $\mathrm{SU}$ peak is observed. For repulsive $\left(Q_{\text {edge }}>0\right)$ charges, SU are formed but very small in magnitude. The highest SU peaks are formed for weakly bound donor charges $\left(Q_{\text {edge }}<0\right)$, which attract the holes of $\mathrm{X}^{+}$, marked with a star in the figure. As in the $\mathrm{X}^{-}$case, if the attractive charge further increases it 
starts dissociating the trion. Consequently, SU peaks are quenched again. Notice however energy splittings for $\mathrm{X}^{+}$(Fig. S2) are smaller than for $\mathrm{X}^{-}$(Fig. 1d in the main text). This is expected from the heavier masses of holes.

\section{Effect of charge impurity location}
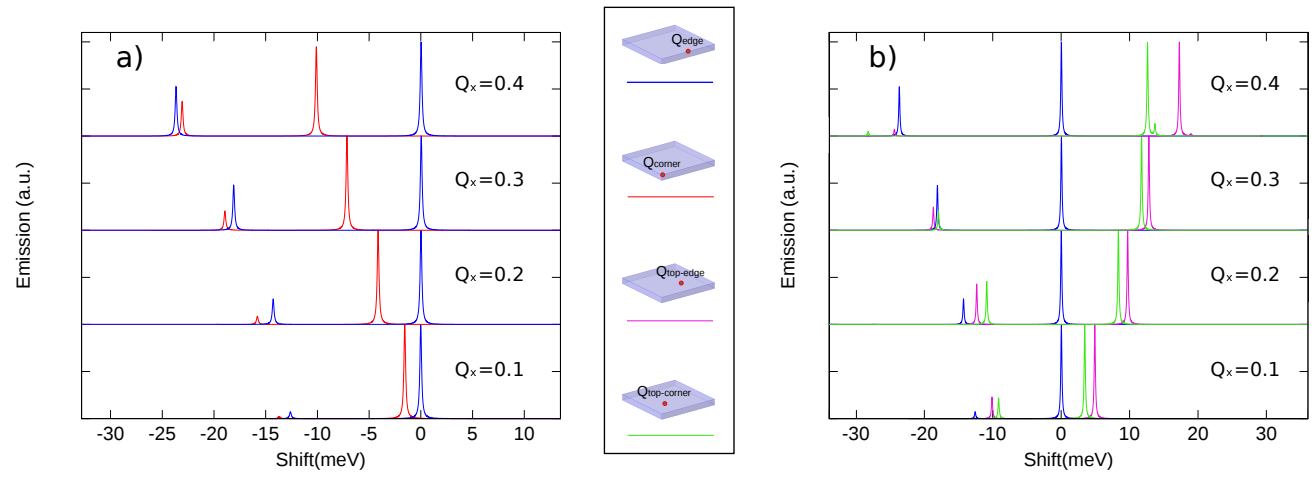

Figure S3: $\mathrm{X}^{-}$emission spectra for different locations of surface charges. The spectra are normalized with respect to the energy and intensity of the $Q_{e d g e}$ fundamental transition. (a) Edge-located vs. corner-located impurity. Blue and red lines stand for edge and corner, respectively. (b) Edge-located vs. edge-top-located vs. corner-top-located. Blue, green and pink lines stand for edge, top-corner and top-edge, respectively. $n e=n h=12$.

In the main text we present the representative cases of a surface charge centered on top of the NPL $\left(Q_{t o p}\right)$, and that of a charge on the edge of lateral sidewall $\left(Q_{\text {edge }}\right)$. In Figure S3 we compare with different locations. One can see that the effect of a charge located in the corner, red line in Fig. S3a, provides similar SU peaks to that of the edge charge, blue line in the figure, both in energy and intensity. We recall that these traps seem to be particularly likely according to recent studies on ligand desorption. ${ }^{6,7}$ Off-centered charges on top and bottom surfaces are studied in Fig. S3b. They give rise to SU peaks of similar height to that of $Q_{e d g e}$, although they reach the optimal charge value sooner than $Q_{e d g e}$ $\left(Q_{\text {top-edge }} \sim Q_{\text {top-corner }} \approx 0.2\right.$ versus $\left.Q_{\text {edge }}=0.4\right)$, because they lie closer to the center of the NPL, where photogenerated carriers tend to localize. 


\section{Effect of conduction band offset in CdSe/CdS NPLs}

The value of the CdSe/CdS conduction band offset (CBO) has been a subject of debate in nanocrystal heterostructures. ${ }^{8-10}$ We used, along our main text, an upper-bound unstrained value of $0.48 \mathrm{eV},{ }^{8}$ which is partly reduced by compressive strain in the core. ${ }^{10}$ Here we explore the scenario where we use a lower-bound ${ }^{9}$ value as well, to see the possible effect of enhancing electron delocalization over the CdS shell. Figure S4 compares the two cases. Lowering the $\mathrm{CBO}$ gives rise to slightly weaker electron-electron repulsion $\left(V_{e e}\right)$ and electronhole attraction $\left(V_{e h}\right)$, however the differences are very small. One can then expect similar role of SU processes as in the main text.
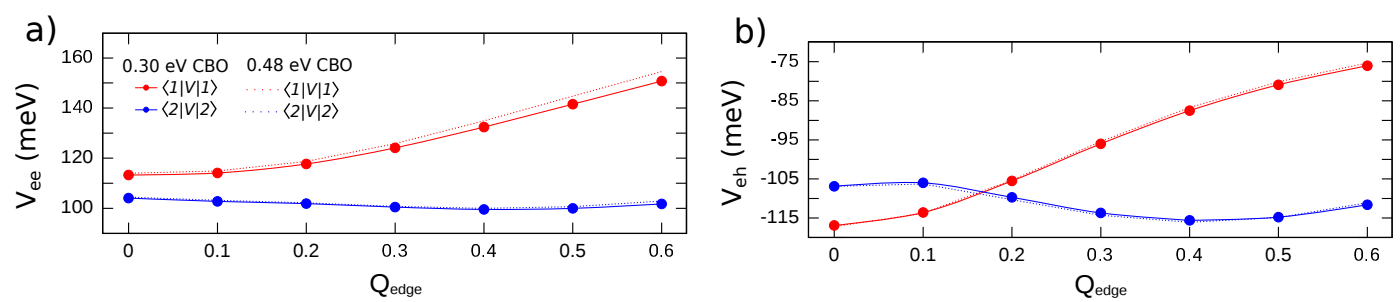

Figure S4: (a,b) Average Coulomb integrals for $Q_{\text {edge }}=0.6$ : (a) electron-electron repulsion and (b) electron-hole attraction for every CBO. $n e=n h=22$ 


\section{Effect of shell thickness in CdSe/CdS NPLs}

Along the main text, core/shell NPLs under study had a shell thickness of 6ML on each side of the core. The experiments of Antolinez et al. ${ }^{11}$ however used thicker shells (12 ML). In this section we compare qualitatively the response in the two cases using a moderate basis set $(n e=n h=12$ ), which permits addressing the experimental dimensions without the computational burden of the large basis set (for $12 \mathrm{ML}$ thickness, the extended CI computation is beyond our current resources).

a)

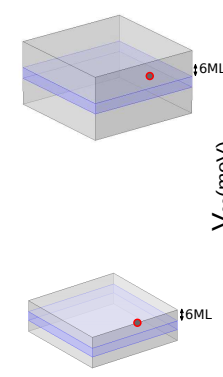

b)

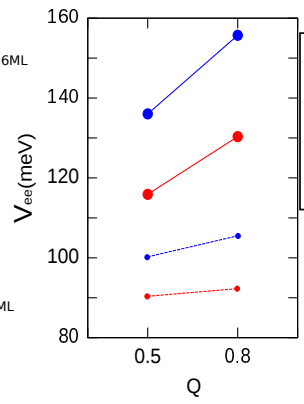

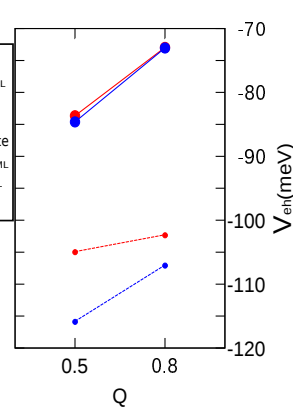

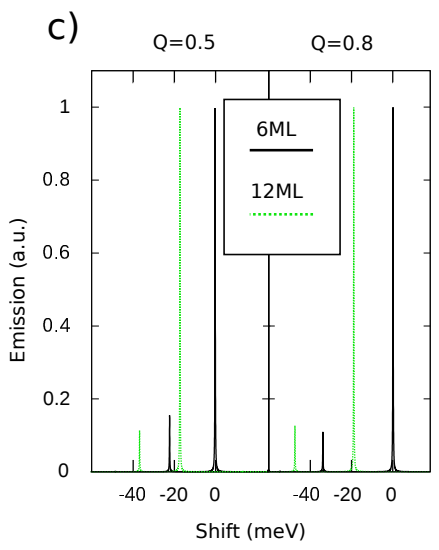

Figure S5: (a) Sketch of the NPLs we are comparing: 12ML shell (top) and 6ML (bottom). The charge is located at same coordinates. (b,c) Coulomb interactions: (b) repulsions e-e and (c) attractions e-h for $Q=0.5$ and $Q=0.8$. (d,e) Normalized emission spectra of 6ML vs 12ML: (d) $Q=0.5$ and (e) $Q=0.8 ; Q=0$ is centred at band edge recombination energy for $6 \mathrm{ML}$ in both cases. $n e=n h=12$

If we focus on the charge location in both systems, Fig. S5a, one may expect similar behaviour. The main difference, as can be seen in Fig. S5b (left panel) occurs for repulsive electron-electron interactions, which are slightly weaker for thick shells. This is a consequence of the larger electron delocalization, which translates into smaller $\left|c_{2}\right|$ coefficients in the CI expansion (see main text) and hence slightly smaller SU satellite, as observed in Fig. S5c. 


\section{Effect of inserting multiple impurities in CdSe/CdS}

We consider here the possibility that two surface traps, instead of one, are acting as electrostatic impurities in CdSe/CdS NPLs. Since there is a general preference of forming defects in the heterostructure interfaces - because of lattice mismatch ${ }^{10,12}$ - and on lateral facets - where ligand desorption is more likely to happen ${ }^{6}$-, we choose the charges to be located as shown in Fig. S6a. The presence of two charges, combined with the weak in-plane confinement, easily dissociates the trion by driving one electron to each surface impurity. This can be seen in the charge densities of Fig. S6b. The number of visible SU peaks, however, remains one (see Fig. S6c). In the case of strong surface charges $(Q=1.0)$, the trion triplet (discussed in the main text) becomes so close in energy to the singlet ground state that it shows up in the spectrum at $4 \mathrm{~K}$, see right panel in Fig. S6c.

a)

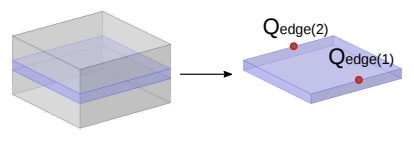

b)

\begin{tabular}{|c|c|c|}
\hline \begin{tabular}{c}
$Q_{\text {edge }}$ \\
\hline $\begin{array}{c}\text { Electrons } \\
(X)\end{array}$
\end{tabular} & 0.5 & 1.0 \\
\hline $\begin{array}{c}\text { Hole } \\
\left(X^{\prime}\right)\end{array}$ & $\mathbf{O}$ & $\mathbf{0}$ \\
\hline
\end{tabular}

c)

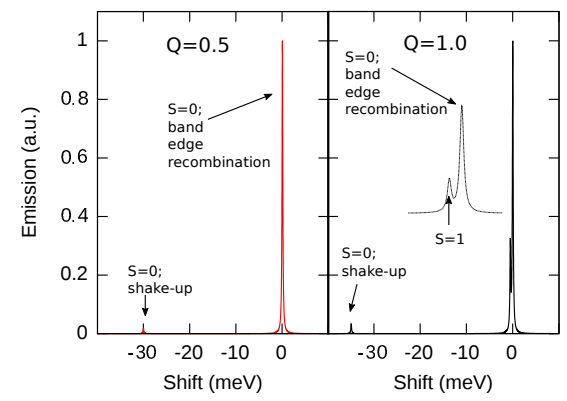

Figure S6: (a) Schematic of a CdSe/CdS NPL with 2 charges on edges intersecting interface and sidewall facet. $Q_{\text {edge(1) }}=Q_{\text {edge(2) }}=Q_{\text {edge}}$. The NPL shell is 12 ML thick. (b) In-plane electrons and hole charge densities for the $\mathrm{X}^{-}$singlet $(S=0)$ ground state at $Q=0.5$ and $Q=1.0$; (c) Normalized emission spectra at $Q=0.5$ (left) and $Q=1.0$ (right). $n e=n h=12$.

If we further increase the charge $Q$ (e.g. by assuming double point charges on each side of the NPL, see Fig. S7a), additional peaks start showing up in the emission spectrum, which is shown in Fig. S7g. The sketches in Fig. S7d-f assign each peak to a corresponding recombination process. Two transitions come from the $\mathrm{X}^{-}$singlet ground state, namely its band edge $(s-R 1)$ and first $\mathrm{SU}(s-S U)$ recombinations. The other transitions are fully radiative recombinations arising from the triplet state, $t-R 1$ and $t-R 2$. The picture is analogous to 
that proposed in the Discussion section of the main text to explain the multi-peaked emission of ref., ${ }^{11}$ but in this case the triplet state is thermally occupied at $4 \mathrm{~K}$, so there is no need to assume slow spin relaxation. The top panel in Fig S7g qualitatively resembles the clusters of four peaks often observed by Antolinez and co-workers in their photoluminescence measurements, ${ }^{11}$ although the inter-peak energy splittings here are one order of magnitude smaller. As mentioned in the main text, assuming the triplet state is metastable even if it is beyond $k T$ from the singlet ground state, and varying trapped charge location, it may be possible to retrieve the experimental spectra.

a)

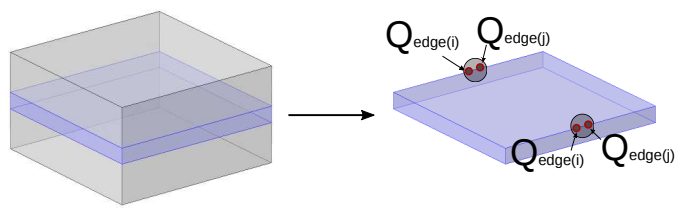

b)

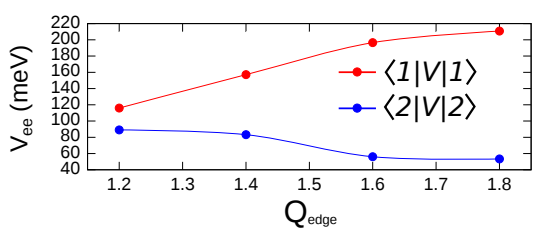

c)

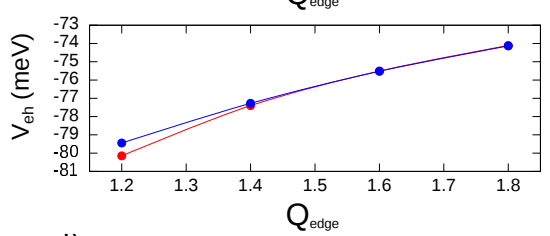

g)

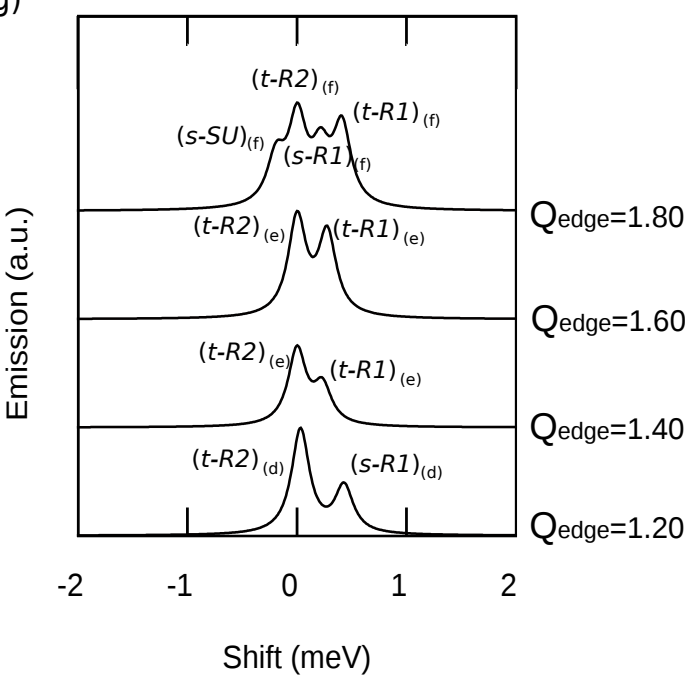

d)

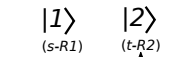
$\mathrm{A}^{\prime} \stackrel{(s-R 1)}{\mathrm{A}^{\prime}+\frac{(t-R 2)}{\uparrow}} \mathrm{A}^{\prime}$

$\mathrm{A}^{\prime} \stackrel{\mathrm{I}}{\mathrm{A}} \mathrm{A}^{\prime}$ band edge

e)

$|1\rangle(t-R 2)$

$A_{A^{\prime}:}:|2\rangle(t-R 2)$

$A^{\prime}$

band edge

recombination

f)

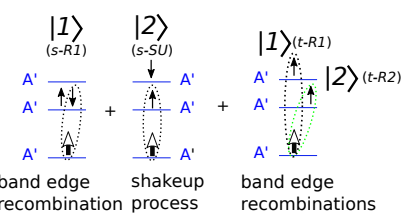

Figure S7: (a) Schematic of a CdSe/CdS NPL with 2 double charges on edges intersecting interface and sidewall facet. (b,c) Coulomb interactions: (b) electron-electron repulsion and (c) electron-hole attraction for configurations $|1\rangle$ and $|2\rangle$. (d-f) Recombination processes involved in each transition. (g) Normalized emission spectrum. The energy origin is set at the position of the brightest peak, $t-R 2$. $Q_{\text {edge }}$ is the net charge on each edge (times the fundamental electron charge). $n e=n h=12$. 


\section{Effect of lateral shape}

In the main text we study NPLs with squared geometry because we model the NPLs of ref. ${ }^{11}$ experiments. Since symmetry lowering opens channels for SU processes, we wish to study if usual shape deviations -towards rectangular geometry- prompt additional SU lines. In Fig. S8 we compare the calculated emission spectrum for squared and rectangular CdSe/CdS NPLs. In the absence of surface charges, Figs. S8a and S8b, none of the two structures displays SU peaks. The reason is that symmetry lowering does not suffice to yield SU peaks. As shown in Fig. 2f,g of the main text, one also needs to enhance repulsion between the two excess carriers and reduce attraction with the opposite charge carrier to stimulate Coulomb admixture with $\left|2_{X^{-}}\right\rangle$for the trion ground state. This was observed for example in type-II, rectangular shaped, core/crown CdSe/CdTe NPLs. ${ }^{13}$ It can be achived in type-I and quasitype-II NPLs with trap charges, as they lower the symmetry and partly dissociate the trion. Figs. S8c and S8d show that the presence of a lateral charge gives rise to a sizable SU peak in both squared and rectangular NPLs. In the case of the rectangular NPL, the SU peak is relatively smaller than in the squared one because of the stronger lateral confinement, which reduces Coulomb admixture. Figure S8 shows that deviations from perfect square geometry in core/shell CdSe/CdS NPLs do not induce additional SU peaks, thus reinforcing the belief that additional factors - other than SU processes- are needed to explain the observation of 3-4 emission peaks in ref. ${ }^{11}$ experiments. 


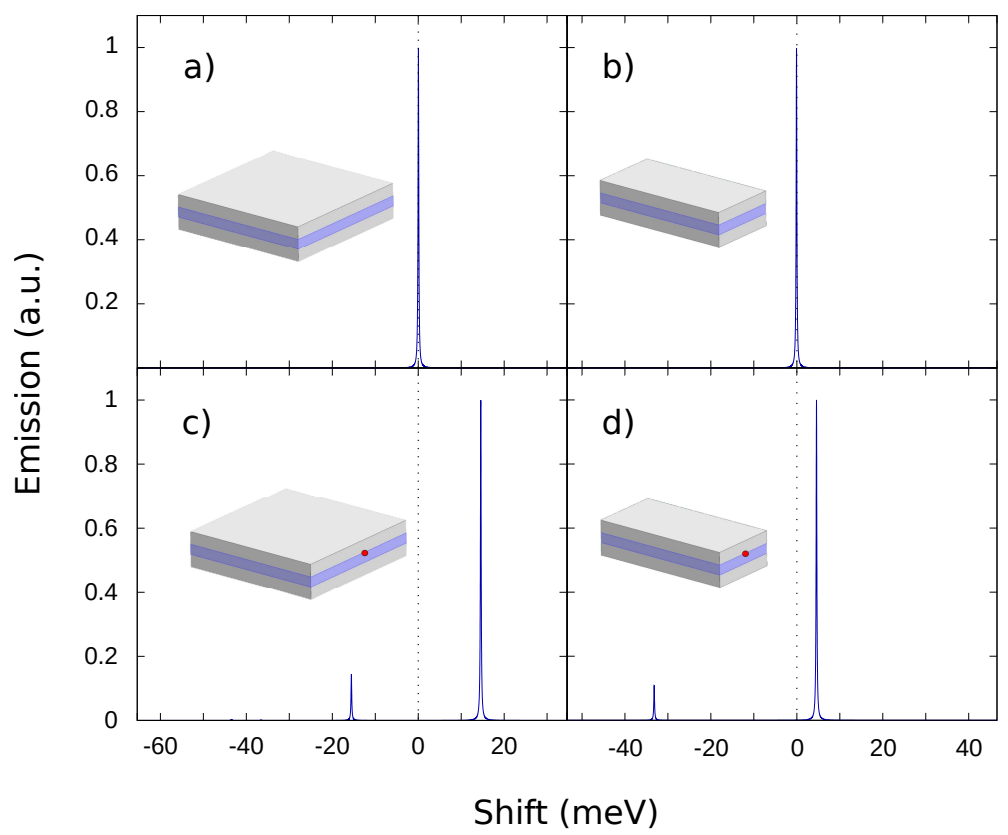

Figure S8: Normalized emission spectrum of $\mathrm{X}^{-}$in squared $(\mathrm{a}, \mathrm{c})$ and rectangular $(\mathrm{b}, \mathrm{d})$ CdSe/CdS NPLs. ( $\mathrm{a}, \mathrm{b})$ show the spectra for $Q_{\text {edge }}=0.0$ (no surface charge), $(\mathrm{c}, \mathrm{d})$ for $Q_{\text {edge }}=$ 0.6. Squared (rectangular) NPLs have $20 \times 20 \mathrm{~nm}^{2}\left(20 \times 10 \mathrm{~nm}^{2}\right)$ in-plane dimensions. The energy origin is set at the position of the brightest peak of the uncharged NPL. $n e=n h=12$.

\section{References}

(1) Jacak, L.; Hawrylak, P.; Wojs, A. Quantum Dots. Springer 1998

(2) Rontani, M.; Cavazzoni, C.; Bellucci, D.; Goldoni, G. Full configuration interaction approach to the few-electron problem in artificial atoms. J. Chem. Phys. 2006, 124, 124102.

(3) Rontani, M.; Eriksson, G.; Åberg, S.; Reimann, S. On the renormalization of contact interactions for the configuration-interaction method in two-dimensions. J. Phys. B 2017, 50, 065301.

(4) Shumway, J.; Franceschetti, A.; Zunger, A. Correlation versus mean-field contributions to excitons, multiexcitons, and charging energies in semiconductor quantum dots, Phys. Rev. B 2001, 63, 155316.

(5) Rajadell, F.; Climente, J.I.; Planelles, J. Excitons in core-only, core-shell and core- 
crown CdSe nanoplatelets: Interplay between in-plane electron-hole correlation, spatial confinement, and dielectric confinement. Phys. Rev. B 2017, 96, 035307.

(6) Leemans, J.; Singh, S.; Li, C.; Ten Brinck, S.; Bals, S.; Infante, I.; Moreels, I.; Hens, Z. Near-Edge Ligand Stripping and Robust Radiative Exciton Recombination in CdSe/CdS Core/Crown Nanoplatelets. J. Phys. Chem. Lett. 2020, 11, 3339-3344.

(7) Drijvers, Emile; De Roo, Jonathan; Martins, Jose C.; Infante, Ivan; Hens, Zeger, Ligand displacement exposes binding site heterogeneity on CdSe nanocrystal surfaces, Chemistry of Materials 2018, 30, 1178-1186.

(8) Wei, S.H.; Zhang S.B.; Zunger, A. First-principles calculation of band offsets, optical bowings, and defects in CdS, CdSe, CdTe, and their alloys. J. Appl. Phys. 2000, 87, 1304.

(9) Steiner, D.; Dorfs, D.; Banin, U.; Della Sala, F.; Manna, L.; Millo, O. Determination of Band Offsets in Heterostructured Colloidal Nanorods Using Scanning Tunneling Spectroscopy Nano Lett. 2008, 8, 2954-2958.

(10) Llusar, J.; Planelles, J.; Climente, J.I. Strain in Lattice-Mismatched CdSe-Based Core/Shell Nanoplatelets. J. Phys. Chem. C 2019, 123, 21299-21306.

(11) Antolinez F.V.; Rabouw F.T.; Rossinelli A.A.; Cui J., Norris D.J. Observation of Electron Shakeup in CdSe/CdS Core/Shell Nanoplatelets. Nano Lett. 2019, 19, 8495-8502.

(12) Li, Q.; Wu, K.; Chen, J.; Chen, Z.; McBride, J.R.; Lian, T. Size-Independent Exciton Localization Efficiency in Colloidal CdSe/CdS Core/Crown Nanosheet Type-I Heterostructures. ACS Nano 2016, 10, 3843-3851.

(13) Steinmetz, V.; Climente, J. I.; Pandya, R.; Planelles, J.; Margaillan, F.; Puttisong, Y.; Marion, D.; Ithurria, S.; Sharma, A.; Lakhwani, G.; Legrand, L.; Bernardot, F.; Testelin, C.; Chamarro, M.; Chin, A.; Rao, A.; Barisien, T. Emission State 
Structure and Linewidth Broadening Mechanisms in Type-II CdSe/CdTe Core-Crown Nanoplatelets: A Combined Theoretical - Single Nanocrystal Optical Study, The Journal of Physical Chemistry C, 2020, 124, 17352-17363. 